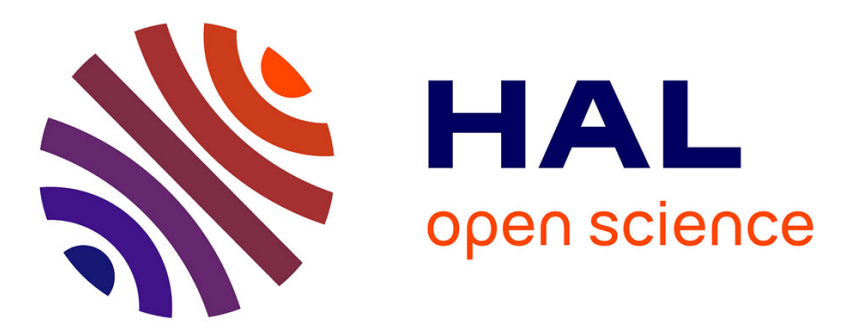

\title{
Ranking of fuzzy intervals seen through the imprecise probabilistic lens
}

Sébastien Destercke, Ines Couso

\section{To cite this version:}

Sébastien Destercke, Ines Couso. Ranking of fuzzy intervals seen through the imprecise probabilistic lens. Fuzzy Sets and Systems, 2015, 278, pp.20-39. 10.1016/j.fss.2014.12.009 . hal-01184644

\section{HAL Id: hal-01184644 \\ https://hal.science/hal-01184644}

Submitted on 17 Aug 2015

HAL is a multi-disciplinary open access archive for the deposit and dissemination of scientific research documents, whether they are published or not. The documents may come from teaching and research institutions in France or abroad, or from public or private research centers.
L'archive ouverte pluridisciplinaire HAL, est destinée au dépôt et à la diffusion de documents scientifiques de niveau recherche, publiés ou non, émanant des établissements d'enseignement et de recherche français ou étrangers, des laboratoires publics ou privés. 


\title{
Ranking of fuzzy intervals seen through the imprecise probabilistic lens
}

\author{
Sébastien Destercke ${ }^{1}$ and Inés Couso ${ }^{2}$ \\ 1. UMR CNRS 7253 Heudyasic UTC, Compiègne, France. \\ 2. Statistics Department, University of Oviedo, Gijón, Spain.
}

\begin{abstract}
Within the fuzzy literature, the issue of ranking fuzzy intervals has been addressed by many authors, who proposed various solutions to the problem. Most of these solutions intend to find a total order on a given collection of fuzzy intervals. However, if one sees fuzzy intervals as descriptions of uncertain quantities, an alternative to rank them is to use ranking rules issued from the imprecise probabilistic literature. In this paper, we investigate ranking rules based on different statistical features (mean, median) and orderings, and relate the obtained (partial) orders to some classical proposals. In particular, we propose a generic expression of stochastic orderings, and then use it to systematically investigate extensions of the most usual stochastic orderings to fuzzy intervals. We also show some relations between those extensions, and explore their relation with existing fuzzy ranking proposals.
\end{abstract}

Keywords: ranking, preferences, partial orders, fuzzy numbers, possibility, decision making

\section{Introduction}

Ranking values or alternatives is an important problem in decision making that has attracted attention in all kinds of fields and domains (multi-criteria decision making [27], machine learning [26], ...), especially when such values or alternatives are characterised by ill-known quantities that are pervaded with uncertainty. A particular domain that has been investigated by many researchers is the one where ill-known quantities to rank are described by fuzzy sets.

The problem of fuzzy ranking, i.e., of deciding whether a fuzzy quantity (i.e., a quantity described by a fuzzy set) is larger than another, has indeed generated a rich literature over the years. Many authors have proposed many different ways of comparing fuzzy numbers, with different requirements and intuition supporting the proposals. However, a striking feature is that the majority of the methods to compare and rank

Email addresses: couso@uniovi.es, sdestercke@gmail. com (Sébastien Destercke ${ }^{1}$ and Inés Couso $^{2}$ ) 
fuzzy quantities result in a single number and/or in a complete ordering (the early possibilistic method by Dubois and Prade [22] is a noticeable exception). Some of the rankings described in this paper also result in a single number between 0 and 1 (the lower probability of the event $X>Y$, for instance), and other ones result in a total ordering (the preference criterion based on the lower expectations, $\underline{\mathbb{E}}(X) \geq \underline{\mathbb{E}}(Y)$, for instance).

However, when fuzzy quantities are used to describe some imprecisely or vaguely known quantity (as is often the case when having to rank those quantities), another sensible approach would be to include incomparability in the picture, possibly leading to accept partial rather than precise rankings between fuzzy sets or to summarize fuzzy comparisons as intervals (or more complicated structures) rather than just a single value. This is also coherent with a view seeing fuzzy sets as extended intervals, possibility distributions, random sets or as imprecise probabilistic models in general.

Note that we do not claim by any means that considering incomparability when comparing fuzzy sets is always a desirable feature, yet we think that it may be useful in a number of situations: when the attitude of the decision maker is unknown, or when it is undesirable to add it to the analysis; when uncertain accuracy (e.g., due to uncertainty in the data features) leads one to extract an ensemble of best classifiers or regression models; or simply in situations in which being cautious about what can be said and about possible inferences is desirable.

While some recent works have explored different extensions of classical probabilistic comparison methods to sets of probabilities (see, for instance [10, 19, 31]), only some of them ( [10, 16, 32]) have marginally considered the case of fuzzy sets/possibility distributions in general and of fuzzy intervals in particular. Studying the applications of the different extensions to the fuzzy interval case and relating them to previously proposed ranking in the fuzzy literature is the main topic of this paper. There are at least two reasons to focus on the specific case of fuzzy intervals: they are by far the most commonly encountered type of fuzzy sets in applications, and the simplicity of their mathematical structure allow for an exposition focusing on interpretation rather than on mathematical subtleties.

In this paper, we see fuzzy intervals as imprecise probabilistic models, and we interpret fuzzy ranking or comparison as a 2-step procedure:

1. given two fuzzy intervals modelling our knowledge about two quantities $X, Y \in$ $\mathbb{R}$, we first build a joint model over the product space $\mathbb{R}^{2}$, assuming some (in)dependence;

2. the fuzzy comparison is then built as the evaluation of the lower/upper expectation of some function defined over $\mathbb{R}^{2}$.

Section 2 introduces how fuzzy sets are interpreted in this study, together with how joint information about the variables can be built in this setting. Section 3 recalls the different means to compare intervals, and how to generalize them to stochastic orderings in a very general way [10]. Section 4 then applies these tools to extend the classical stochastic orderings that are statistical preference, statistical dominance and expectation comparisons, to fuzzy intervals. Section 5 then reviews some of the most classical fuzzy ranking methods under the light of imprecise probabilistic approaches. Section 6 then illustrates the proposed notions on some examples similar to those proposed by 
Bortolan and Degani's [5]. Note that a number of ideas pursued in this paper already appeared in section 5.2.2. and 5.2.3. of Dubois [21], although in a preliminary form and with less details than in the present study, hence a number of our results can be seen as an elaboration of these ideas. We also complement Dubois preliminary proposals by providing a general expression for orderings (Section 3.2), by studying other notions of comparisons (i.e., weak ordering, maximin and maximax), by studying the relations between the different (partial) rankings, and by precisely linking our different proposals to previously proposed fuzzy ranking methods.

Shortly speaking, the main contributions of this paper are two fold:

1. We study the problem of fuzzy ranking from an imprecise probabilistic perspective, therefore introducing new ways to rank fuzzy intervals (some of them including incomparability statements). In the case where fuzzy intervals describe uncertain quantities, this allows us to build well-funded rankings extending classical statistical and interval comparisons, that usually come with clear interpretations and assumptions.

2. We relate some classical fuzzy rankings to the newly introduced rankings. This allows us to shed new lights on those rankings, and provides them with new interpretations (possibly justifying their use in new settings).

\section{Fuzzy sets as imprecise probabilities and random sets}

We consider the problem of comparing two fuzzy sets expressed by their equivalent possibility distributions (or membership functions) $\pi_{X}$ and $\pi_{Y}$. We consider that these fuzzy sets model our uncertainty about values, that is we adopt the epistemic view [11]. There are at least two possible interpretations about these values. According to the first interpretation, we assume that they represent imprecise information about a pair of fixed but unknown values, for example $\pi_{X}, \pi_{Y}$ may describe the weight and height of an individual, which may be ill-known due to the use of a poor balance scale, but are nevertheless fixed. According to the second interpretation, we assume that $X, Y$ are random quantities, and that their respective probability distributions are dominated by the possibility measures derived from $\pi_{X}$ and $\pi_{Y}$. For example, $\pi_{X}$ and $\pi_{Y}$ may describe our limited knowledge about the distribution of a population. Formally:

$$
P_{X}(A) \leq \sup _{x \in A} \pi_{X}(x), P_{Y}(B) \leq \sup _{y \in B} \pi_{Y}(y), \forall A, B \in \beta_{\mathbb{R}},
$$

where $\beta_{\mathbb{R}}$ denotes the usual Borel $\sigma$-field on the real line. Results and rankings presented within this paper better fit with the second interpretation, but some of them could fit with the first one.

In this paper, to reduce mathematical complexity to the benefits of intuition, we will assume that fuzzy sets $\pi$ are fuzzy intervals (note that it is the case in most works on fuzzy rankings, see [42, 21] and references within). Recall that the distribution $\pi_{X}: \mathbb{R} \rightarrow[0,1]$ of a fuzzy interval is such that for all $a, b, c \in \mathbb{R}$ such that $a \leq b \leq c$, we have $\pi_{X}(b) \geq \min \left(\pi_{X}(a), \pi_{X}(c)\right)$. We will also restrict the study to normalized distributions for which there is a value $x \in \mathbb{R}$ such that $\pi_{X}(x)=1$ (otherwise the set of probabilities dominated by $\pi_{X}$ becomes empty). 


\subsection{Marginal fuzzy sets}

Let us first recall some necessary notions about possibility distributions. Given a possibility distribution $\pi: \mathbb{R} \rightarrow[0,1]$ describing a fuzzy interval, recall that we can define:

- Possibility ( $\Pi$ ) and necessity $(N)$ measures over any set $A \subseteq \mathbb{R}$, defined as

$$
\Pi(A)=\sup _{x \in A} \pi(x) ; \quad N(A)=1-\Pi\left(A^{c}\right)=\inf _{x \in A^{c}}(1-\pi(x))
$$

where $A^{c}$ is the complement of $A$.

- A (weak) alpha cut $\pi_{\alpha}$ for each $\left.\left.\alpha \in\right] 0,1\right]$ such that

$$
\pi_{\alpha}=\{x \in \mathbb{R} \mid \pi(x) \geq \alpha\}
$$

and we will denote $\underline{\pi}_{\alpha}=\inf \left\{x \mid x \in \pi_{\alpha}\right\}, \bar{\pi}_{\alpha}=\sup \left\{x \mid x \in \pi_{\alpha}\right\}$ the upper and lower bounds of these $\alpha$-cuts, respectively. A strong alpha cut $\pi_{\alpha}^{>}$for each $\alpha \in$ $[0,1[$ such that

$$
\pi_{\alpha}^{>}=\{x \in \mathbb{R} \mid \pi(x)>\alpha\} .
$$

- A set of probabilities $\mathscr{P}(\Pi)$ bounded by $\Pi$ such that

$$
\mathscr{P}(\Pi)=\left\{P \in \mathscr{P}_{\beta_{\mathbb{R}}} \mid P(A) \leq \Pi(A) \forall A \in \beta_{\mathbb{R}}\right\}
$$

with $\mathscr{P}_{\beta_{\mathbb{R}}}$ the set of all probabilities on the usual Borel $\sigma$-field of the real line.

Alternatively, a possibility distribution $\pi$ can also be described by an equivalent random set $\Gamma_{\pi}$ that maps the unit interval (provided with the uniform distribution $\mathscr{U}_{[0,1]}$ ) to the alpha-cuts, i.e. $\Gamma_{\pi}:[0,1] \rightarrow \mathbb{R}$ with $\mathbb{R}$ the intervals on $\mathbb{R}$ and $\Gamma_{\pi}(\alpha)=\pi_{\alpha}$. We then have

$$
\Pi(A)=P_{\mathscr{Q}[0,1]}\left(\left\{\alpha \mid \Gamma_{\pi}(\alpha) \cap A \neq \emptyset\right\}\right) .
$$

This equation is simply a rewriting of Equation 1 in terms of random sets, and the two models describe the same set $\mathscr{P}(\Pi)$ of dominated probabilities.

Other tools that will be used in this study are the notions of p-boxes [23] and of lower/upper expectations (also commonly called lower and upper previsions [41]). A p-box $[\underline{F}, \bar{F}]$ is a pair of cumulative distributions such that $\underline{F}(x) \leq \bar{F}(x)$ for all $x \in \mathbb{R}$. The p-box $\left[\underline{F}_{\pi}, \bar{F}_{\pi}\right]$ associated to a fuzzy set $\pi$ is the pair of cumulative distributions such that

$$
\underline{F}_{\pi}(x)=N((-\infty, x]) \quad \text { and } \quad \bar{F}_{\pi}(x)=\Pi((-\infty, x]), \forall x \in \mathbb{R} .
$$

A fuzzy interval and its associated p-box are pictured in Figure 1

Similarly, to a fuzzy interval described by distribution $\pi_{X}$, we can associate lower and upper expectation bounds $\underline{\mathbb{E}}_{\pi_{X}}(\mathrm{id})$ and $\overline{\mathbb{E}}_{\pi_{X}}$ (id) reached within the set $\mathscr{P}(\Pi)$. Such bounds are simply expressed as

$$
\underline{\mathbb{E}}_{\pi_{X}}(\mathrm{id})=\int_{\mathbb{R}} x d \bar{F}_{\pi}(x)=\int_{0}^{1} \underline{\pi}_{\alpha} d \alpha \quad \text { and } \quad \overline{\mathbb{E}}_{\pi_{X}}(\mathrm{id})=\int_{\mathbb{R}} x d \underline{F}_{\pi}(x)=\int_{0}^{1} \bar{\pi}_{\alpha} d \alpha .
$$




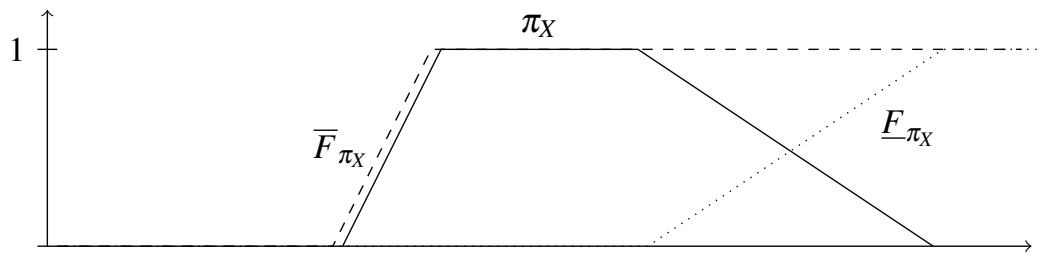

Figure 1: A fuzzy interval and its associated p-box

(In the last expressions, id : $\mathbb{R} \rightarrow \mathbb{R}$ represents the identity function, $\operatorname{id}(x)=x, \forall x \in$ $\mathbb{R})$. Also note that, given a function $f$ on $\mathbb{R}$, the lower and upper expectations of this function within $\mathscr{P}(\Pi)$ read

$$
\underline{\mathbb{E}}_{\pi_{X}}(f)=\int_{0}^{1} \inf _{x \in \pi_{\alpha}} f(x) d \alpha \quad \text { and } \quad \overline{\mathbb{E}}_{\pi_{X}}(f)=\int_{0}^{1} \sup _{x \in \pi_{\alpha}} f(x) d \alpha .
$$

The lower and upper expectation bounds are nothing else but the lower and upper extremes of the "mean value" of the fuzzy interval $X$ defined by Dubois and Prade [20].

According to [13], a distribution $\pi$ represents the family of probability measures satisfying the following inequalities w.r.t. the strong alpha cuts:

$$
P\left(\pi_{\alpha}^{>}\right) \geq 1-\alpha, \forall \alpha \in(0,1] .
$$

Such a family is in fact the family of probability measures that are bounded between the necessity measure $N$ and the possibility measure $\Pi,\left\{P: P(A) \geq N(A), \forall A \in \beta_{\mathbb{R}}\right\}=$ $\left\{P: P(A) \leq \Pi(A), \forall A \in \beta_{\mathbb{R}}\right\}$.

\subsection{Joint models from marginal fuzzy sets}

In the imprecise probabilistic literature, there are multiple ways to define (in)dependence (e.g., see [9, 14]). In this paper, we will not deal with all of them, and will concentrate mainly on notions easily expressible through $\alpha$-cuts (such as Zadeh's extension [47]) and random sets associated to fuzzy intervals.

In this work, we will assume mainly three kinds of dependence relations between two distributions $\pi_{X}$ and $\pi_{Y}$, as these dependencies will allow us to connect our results to standard fuzzy ranking methods. A convenient way to describe such dependency is by a joint random function $\Gamma_{X Y}:[0,1]^{2} \rightarrow \mathbb{R}^{2}$ mapping each point $(\alpha, \beta)$ of $[0,1]^{2}$ to the Cartesian product $\pi_{X, \alpha} \times \pi_{Y, \beta}$ and a specific joint probability measure $P$ defined over the initial space $[0,1]^{2}$.

- monotonic dependence (M), that assumes a totally positive correlation between alpha-cuts, in this case the initial $P$ is the uniform distribution on the line $\alpha=\beta$ of $[0,1]^{2}$. This is nothing else but the application of Zadeh's extension [47] principle. The result is then a joint distribution $\pi_{X Y}$ such that $\pi_{X Y, \alpha}=\pi_{X, \alpha} \times$ $\pi_{Y, \alpha}$. This is the only dependence assumption whose joint random sets are nested and therefore the only joint model to be again a fuzzy set/possibility distribution. This is why it is usually the one considered in fuzzy set theory; 
- random set independence (I) where $P=\mathscr{U}_{[0,1]} \otimes \mathscr{U}_{[0,1]}$ is the stochastic product of the two original uniform distributions;

- anti-monotonic dependence (AM) where totally negative correlation between alpha-cuts is assumed. In this case the initial probability measure $P$ is the uniform distribution on the line $\alpha=1-\beta$ of $[0,1]^{2}$. This dependence relation can be represented by means of a random set that maps each value $\alpha$ to the Cartesian product $\pi_{X, \alpha} \times \pi_{Y, 1-\alpha}$, where the uniform distribution is defined on $[0,1]$. This is also equivalent to assume monotonic dependence between $\pi_{X}$ and $1-\pi_{Y}$. It should be noted that, similarly to what happens with the lower Frechet Bounds [4], this notion of dependence cannot be applied in a pair-wise way to three different variables, yet the consideration of such dependencies makes sense in some settings (e.g., see [17] for its use in statistical preferences).

In Section 4, we will see that the choice of a specific (in)dependence notion actually only matters when we consider the extension of the notion of statistical preference to possibility distributions.

\section{Interval comparisons and stochastic orderings}

In this section, we first recall classical ways to compare intervals (Subsection 3.1), as well as the generic framework proposed by Couso and Dubois [10] (Subsection 3.2) to extend stochastic orderings to probability sets. This is the occasion to explore some particular properties satisfied by this framework in our particular case, and to introduce specific notations used in the sequel.

\subsection{Interval comparisons}

When two values $X=[\underline{x}, \bar{x}]$ and $Y=[y, \bar{y}]$ are described by intervals, the classical comparison between numbers can be extended into several ways, either by choosing specific points within the intervals as representatives, or by admitting that some intervals may be incomparable due to imprecision.

Rules of the first family include:

- Weak ordering: $X \leq_{w o} Y$ if $\underline{x} \leq \bar{y}$

- Maximin [38, 40]: $X \leq_{M m} Y$ if $\underline{x} \leq \underline{y}$

- Maximax [35]: $X \leq_{M M} Y$ if $\bar{x} \leq \bar{y}$

- Hurwicz [29]: $X \leq_{H(\gamma)} Y$ if $\gamma \underline{x}+(1-\gamma) \bar{x} \leq \gamma \underline{y}+(1-\gamma) \bar{y}$ where $\gamma \in[0,1]$.

If $X$ and $Y$ are interpreted as possible rewards (corresponding to the accuracy of a classifier, to the benefits of some choice/policy, ...), then maximin is usually associated to a pessimistic attitude (focusing on the lowest possible reward) and maximax to an optimistic attitude. The parameter $\gamma$ can then be interpreted as an index of pessimism, since maximin is retrieved when $\gamma=1$, and maximax when $\gamma=0$. Given any two intervals $X$ and $Y$, the above criteria will always result in a comparison: either $X \leq Y$, 


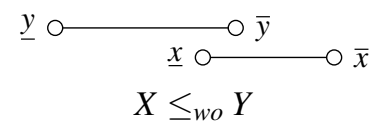

a. Weak ordering

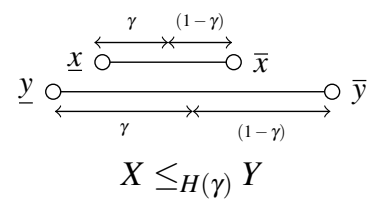

d. Hurwicz ordering

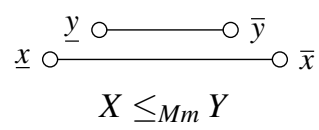

b. Maximin

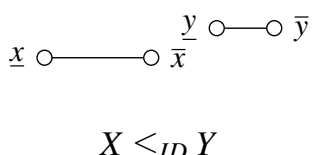

e. Interval dominance

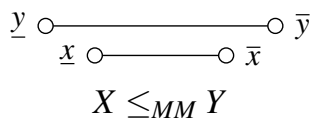

c. Maximax

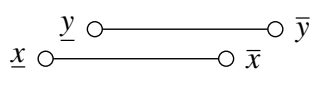

$X \leq_{L o} Y$

f. Lattice ordering

Figure 2: Interval comparisons illustrations

$Y \leq X$, or both (in case of indifference). That is, given a set $X_{1}, \ldots, X_{N}$ of intervals, the above rankings will always provide a complete (pre-)order.

On the contrary, the two following extensions usually produce partial orders between intervals:

- Interval dominance [24]: $X \leq_{I D} Y$ if $\bar{x} \leq \underline{y}$

- lattice ordering: $X \leq_{L o} Y$ if $\underline{x} \leq \underline{y}$ and $\bar{x} \leq \bar{y}$

Interval dominance says that two intervals are incomparable as soon as they overlap (it produces so-called interval orders), while the lattice ordering is the canonical order induced by the lattice of intervals (i.e. $X \leq_{L o} Y$ iff $\sup (X, Y)=Y$, or equivalently iff $\inf (X, Y)=X)$. Note that when $X, Y$ are intervals, the following implications between the above orderings hold:

$$
X \leq_{I D} Y \Rightarrow X \leq_{L o} Y \Rightarrow\left\{\begin{array}{c}
X \leq_{M m} Y \\
X \leq_{M M} Y \\
X \leq_{H(\gamma)} Y \text { for any } \gamma \in[0,1]
\end{array}\right\} \Rightarrow X \leq_{w o} Y .
$$

In the next sections, we will apply these interval comparisons to the extensions of well-known stochastic orderings (namely, statistical preference, first stochastic dominance and expectation dominance) to the context of joint possibility distributions. For each combination interval comparison + stochastic ordering, we will obtain a particular ranking (possibly different for different independence assumptions about $X$ and $Y)$. Before that, we introduce a generic way to express stochastic preferences, directly inspired by some previous work of one of the authors [10].

\subsection{Generic expression of stochastic orderings}

Every stochastic ordering in the literature we know of establishes a specific preference relation between pairs $(X, Y)$ of random variables based on their joint probability 
distribution $P_{X Y}$. A well known result in probability theory states that, for any probability measure defined on the Borel $\sigma$-field $\beta_{\mathbb{R}}$, there exists some random variable $X$ defined on the unit interval $[0,1]$, provided with the uniform distribution, that induces it. Thus, without loss of generality, we may assume that the random vector $(X, Y)$ is defined over the unit square $[0,1]^{2}$ and that the marginal distributions of $P$ do coincide with the uniform distribution, $\mathscr{U}_{[0,1]}$.

Several of these stochastic orderings [10] from the literature can be expressed in terms of the expectation of a function $g(X, Y)$ where $g: \mathbb{R}^{2} \rightarrow \mathbb{R}$ can be decomposed as the difference $g(x, y)=f(x, y)-f(y, x)$ where $f$ is increasing in the first component and decreasing in the second one. To facilitate notations, we will denote $f^{s w}(x, y):=$ $f(y, x)$ ( $s w$ standing for swap). According to this general formulation, $X$ will be said to be preferred to $Y$ whenever the expectation of $g(X, Y)$ is non negative. Specific expressions of $f$ (and therefore of $g$ ) then allow one to retrieve classical stochastic orderings.

When considering precise probabilities, the expectation of $g(X, Y)$ being positive is equivalent to the expectation of $f(X, Y)$ being greater than the expectation $f^{s w}(X, Y)$ (this follows from the linearity of precise expectation). However, when considering sets of joint probabilities induced by marginal possibility distributions and a joint model (see Section 2.2, this equivalence is no longer valid, as lower and upper expectations are non-linear operators. Also, the expectations of $g(X, Y), f(X, Y)$ and $f^{s w}(X, Y)$ become intervals instead of numbers.

There are therefore two main ways to extend relations of Section 3.1 by integrating this view of stochastic preferences, given some joint structure $\Gamma_{X Y}$ taken from Section 2.2. The first is to consider the interval $\left[\underline{\mathbb{E}}_{\Gamma_{X Y}}(g), \overline{\mathbb{E}}_{\Gamma_{X Y}}(g)\right]$ given by

$$
\begin{aligned}
& \underline{\mathbb{E}}_{\Gamma_{X Y}}(g)=\int_{[0,1] \times[0,1]} \inf _{x \in \pi_{X, \alpha}, y \in \pi_{Y, \beta}} g(x, y) d P(\alpha, \beta) \text { and } \\
& \overline{\mathbb{E}}_{\Gamma_{X Y}}(g)=\int_{[0,1] \times[0,1]} \sup _{x \in \pi_{X, \alpha}, y \in \pi_{Y, \beta}} g(x, y) d P(\alpha, \beta),
\end{aligned}
$$

where $P$ is the joint probability on the $\alpha, \beta$-cuts, and to compare this interval to the singleton $\{0\}$ using one of the relations of Section 3.1

The second way to blend stochastic ordering expressions and interval comparisons is to compute the intervals $\left[\underline{\mathbb{E}}_{\Gamma_{X Y}}(f), \overline{\mathbb{E}}_{\Gamma_{X Y}}(f)\right]$ and $\left[\underline{\mathbb{E}}_{\Gamma_{X Y}}\left(f^{s w}\right), \overline{\mathbb{E}}_{\Gamma_{X Y}}\left(f^{s w}\right)\right]$ and to compare them using one relation. Values $\underline{\mathbb{E}}_{\Gamma_{X Y}}(f), \overline{\mathbb{E}}_{\Gamma_{X Y}}(f)$ can be computed by

$$
\begin{aligned}
& \underline{\mathbb{E}}_{\Gamma_{X Y}}(f)=\int_{[0,1] \times[0,1]} \inf _{x \in \pi_{X, \alpha}, y \in \pi_{Y, \beta}} f(x, y) d P(\alpha, \beta) \text { and } \\
& \overline{\mathbb{E}}_{\Gamma_{X Y}}(f)=\int_{[0,1] \times[0,1]} \sup _{x \in \pi_{X, \alpha}, y \in \pi_{Y, \beta}} f(x, y) d P(\alpha, \beta) .
\end{aligned}
$$

Values $\underline{\mathbb{E}}_{\Gamma_{X Y}}\left(f^{s w}\right), \overline{\mathbb{E}}_{\Gamma_{X Y}}\left(f^{s w}\right)$ can be derived likewise. According to the following result, there are some noticeable relations between the intervals of expectations of $g(X, Y), f(X, Y)$ and $f^{s w}(X, Y)$.

Proposition 1. Let $f: \mathbb{R}^{2} \rightarrow \mathbb{R}$ be increasing in the first component and decreasing in the second one. Let $g: \mathbb{R}^{2} \rightarrow \mathbb{R}$ be defined from $f$ as $g(x, y)=f(x, y)-f^{s w}(x, y)$. Let $P$ be a probability measure defined on $\beta_{[0,1]^{2}}$, whose respective marginals coincide with the uniform distribution on the unit interval. The following equalities hold:

$$
\text { - } \underline{\mathbb{E}}_{\Gamma_{X Y}}(g)=\underline{\mathbb{E}}_{\Gamma_{X Y}}(f)-\overline{\mathbb{E}}_{\Gamma_{X Y}}\left(f^{s w}\right),
$$


- $\overline{\mathbb{E}}_{\Gamma_{X Y}}(g)=\overline{\mathbb{E}}_{\Gamma_{X Y}}(f)-\underline{\mathbb{E}}_{\Gamma_{X Y}}\left(f^{s w}\right)$,

and therefore we have that

- $\underline{\mathbb{E}}_{\Gamma_{X Y}}(g) \geq 0$ if and only if $\underline{\mathbb{E}}_{\Gamma_{X Y}}(f) \geq \overline{\mathbb{E}}_{\Gamma_{X Y}}\left(f^{s w}\right)$,

- $\overline{\mathbb{E}}_{\Gamma_{X Y}}(g) \geq 0$ if and only if $\overline{\mathbb{E}}_{\Gamma_{X Y}}(f) \geq \underline{\mathbb{E}}_{\Gamma_{X Y}}\left(f^{s w}\right)$.

Proof. Let us check the first equality (the second can be derived in an analogous way). We have that

$$
\underline{\mathbb{E}}_{\Gamma_{X Y}}(g)=\int_{[0,1]^{2}} \inf _{x \in \pi_{X, \alpha}, y \in \pi_{Y, \beta}} g(x, y) d P(\alpha, \beta),
$$

according to Equation 10 Furthermore, according to the properties of $g$, for each $\alpha$ cut, such an infimum can be calculated as follows:

$$
\inf _{x \in \pi_{X, \alpha}, y \in \pi_{Y, \beta}} g(x, y)=g\left(\underline{\pi}_{X, \alpha}, \bar{\pi}_{Y, \beta}\right)=f\left(\underline{\pi}_{X, \alpha}, \bar{\pi}_{Y, \beta}\right)-f\left(\bar{\pi}_{Y, \beta}, \underline{\pi}_{X, \alpha}\right) .
$$

Therefore, according to the properties of the Lebesgue integral, we can write that:

$$
\begin{aligned}
\underline{\mathbb{E}}_{\Gamma_{X Y}}(g) & =\int_{[0,1]^{2}} f\left(\underline{\pi}_{X, \alpha}, \bar{\pi}_{Y, \beta}\right) d P(\alpha, \beta)-\int_{[0,1]^{2}} f\left(\bar{\pi}_{Y, \beta}, \underline{\pi}_{X, \alpha}\right) d P(\alpha, \beta) \\
& =\underline{\mathbb{E}}_{\Gamma_{X Y}}(f)-\overline{\mathbb{E}}_{\Gamma_{X Y}}\left(f^{s w}\right) .
\end{aligned}
$$

These results imply very strong relations between the two extensions, as shown in the next corollary.

Corollary 2. Following the nomenclature used in Proposition 1 , we have:

- $\left[\underline{\mathbb{E}}_{\Gamma_{X Y}}(f), \overline{\mathbb{E}}_{\Gamma_{X Y}}(f)\right] \geq_{I D}\left[\underline{\mathbb{E}}_{\Gamma_{X Y}}\left(f^{s w}\right), \overline{\mathbb{E}}_{\Gamma_{X Y}}\left(f^{s w}\right)\right]$ if and only if the following condition holds:

$$
\underline{\mathbb{E}}_{\Gamma_{X Y}}(g) \geq 0 \text {. }
$$

- $\left[\underline{\mathbb{E}}_{\Gamma_{X Y}}(f), \overline{\mathbb{E}}_{\Gamma_{X Y}}(f)\right] \geq_{w o}\left[\underline{\mathbb{E}}_{\Gamma_{X Y}}\left(f^{s w}\right), \overline{\mathbb{E}}_{\Gamma_{X Y}}\left(f^{s w}\right)\right]$ if and only if the following condition holds:

$$
\overline{\mathbb{E}}_{\Gamma_{X Y}}(g) \geq 0 .
$$

- $\left[\underline{\mathbb{E}}_{\Gamma_{X Y}}(f), \overline{\mathbb{E}}_{\Gamma_{X Y}}(f)\right] \geq_{H(\gamma)}\left[\underline{\mathbb{E}}_{\Gamma_{X Y}}\left(f^{s w}\right), \overline{\mathbb{E}}_{\Gamma_{X Y}}\left(f^{s w}\right)\right]$ if and only if the following condition holds:

$$
\left[\underline{\mathbb{E}}_{\Gamma_{X Y}}(g), \overline{\mathbb{E}}_{\Gamma_{X Y}}(g)\right] \geq_{H(\gamma)}\{0\} .
$$

Note that we can link Corollary2 2 results to the other interval comparisons explored in Section 3.1 through the following equivalences:

$$
\begin{gathered}
\underline{\mathbb{E}}_{\Gamma_{X Y}}(g) \geq 0 \Leftrightarrow\left[\mathbb{E}_{\Gamma_{X Y}}(g), \overline{\mathbb{E}}_{\Gamma_{X Y}}(g)\right] \geq \omega\{0\} \text { for } \omega \in\{M m, I D, L o\} \\
\overline{\mathbb{E}}_{\Gamma_{X Y}}(g) \geq 0 \Leftrightarrow\left[\underline{\mathbb{E}}_{\Gamma_{X Y}}(g), \overline{\mathbb{E}}_{\Gamma_{X Y}}(g)\right] \geq \omega\{0\} \text { for } \omega \in\{w o, M M\}
\end{gathered}
$$


According to this, any comparison using interval $\left[\underline{\mathbb{E}}_{\Gamma_{X Y}}(g), \overline{\mathbb{E}}_{\Gamma_{X Y}}(g)\right]$ is equivalent to another comparison using function $f$. Thus, in the following, we will only concentrate on the second extension using intervals of $f$ functions. Note that the converse is not true, as any comparison

$$
\left[\underline{\mathbb{E}}_{\Gamma_{X Y}}(f), \overline{\mathbb{E}}_{\Gamma_{X Y}}(f)\right] \geq_{k}\left[\underline{\mathbb{E}}_{\Gamma_{X Y}}\left(f^{s w}\right), \overline{\mathbb{E}}_{\Gamma_{X Y}}\left(f^{s w}\right)\right]
$$

with $k \in\{M m, M M, L o\}$ cannot be expressed by a comparison using $g$ expectation bounds.

Let us establish some notations that will make the expression of further results more concise.

- The lower and upper expectations of a function $f(x, y)$ under monotonic dependence will be respectively denoted by $\underline{\mathbb{E}}_{f, M}$ and $\overline{\mathbb{E}}_{f, M}$ :

$$
\begin{aligned}
& \underline{\mathbb{E}}_{f, M}=\int_{0}^{1} \inf _{(x, y) \in \pi_{X, \alpha} \times \pi_{Y, \alpha}} f(x, y) d \alpha=\int_{0}^{1} f\left(\underline{\pi}_{X, \alpha}, \bar{\pi}_{Y, \alpha}\right) d \alpha . \\
& \overline{\mathbb{E}}_{f, M}=\int_{0}^{1} \sup _{(x, y) \in \pi_{X, \alpha} \times \pi_{Y, \alpha}} f(x, y) d \alpha=\int_{0}^{1} f\left(\bar{\pi}_{X, \alpha}, \underline{\pi}_{Y, \alpha}\right) d \alpha .
\end{aligned}
$$

- The lower and upper expectations of $f(x, y)$ under random set independence will be respectively denoted by $\underline{\mathbb{E}}_{f, I}$ and $\overline{\mathbb{E}}_{f, I}$ :

$$
\begin{aligned}
& \underline{\mathbb{E}}_{f, I}=\int_{0}^{1} \int_{0}^{1} \inf _{(x, y) \in \pi_{X, \alpha} \times \pi_{Y, \beta}} f(x, y) d \alpha d \beta=\int_{0}^{1} \int_{0}^{1} f\left(\underline{\pi}_{X, \alpha}, \bar{\pi}_{Y, \beta}\right) d \alpha d \beta . \\
& \overline{\mathbb{E}}_{f, I}=\int_{0}^{1} \int_{0}^{1} \sup _{(x, y) \in \pi_{X, \alpha} \times \pi_{Y, \beta}} f(x, y) d \alpha d \beta=\int_{0}^{1} \int_{0}^{1} f\left(\bar{\pi}_{X, \alpha}, \underline{\pi}_{Y, \beta}\right) d \alpha d \beta .
\end{aligned}
$$

- The lower and upper expectations of $f(x, y)$ under anti-monotonic dependence will be respectively denoted by $\underline{\mathbb{E}}_{f, A M}$ and $\overline{\mathbb{E}}_{f, A M}$ :

$$
\begin{aligned}
& \underline{\mathbb{E}}_{f, A M}=\int_{0}^{1} \inf _{(x, y) \in \pi_{X, \alpha} \times \pi_{Y, 1-\alpha}} f(x, y) d \alpha=\int_{0}^{1} f\left(\underline{\pi}_{X, \alpha}, \bar{\pi}_{Y, 1-\alpha}\right) d \alpha . \\
& \overline{\mathbb{E}}_{f, A M}=\int_{0}^{1} \sup _{(x, y) \in \pi_{X, \alpha} \times \pi_{Y, 1-\alpha}} f(x, y) d \alpha=\int_{0}^{1} f\left(\bar{\pi}_{X, \alpha}, \underline{\pi}_{Y, 1-\alpha}\right) d \alpha .
\end{aligned}
$$

According to the above nomenclature, and under any particular dependence assumption dep $\in\{M, I, A M\}$, we will say that $X$ is $f$-preferred to $Y$ with respect to a particular ordering ord $\in\{w o, M m, M M, H(\lambda), I D, L o\}$ if:

$$
\left[\underline{\mathbb{E}}_{f, \mathrm{dep}}, \overline{\mathbb{E}}_{f, \mathrm{dep}}\right] \geq_{\text {ord }}\left[\underline{\mathbb{E}}_{f s w}, \mathrm{dep}, \overline{\mathbb{E}}_{f}^{s w}, \mathrm{dep}\right]
$$


We will denote it $X \geq_{f \text {,dep,ord }} Y$. Of course, the implications provided by Equation (9) still hold for these comparisons. Before applying the above results to the case of fuzzy intervals, let us study a specific case of $f$ that we will encounter in the sequel. We define a function $f(x, y)$ as $x$-measurable if $f(x, y)=f\left(x, y^{\prime}\right)$ for all $y, y^{\prime} \in \mathbb{R}$. In this case, we will write $f_{1}(x):=f(x, y)$, as the value of $f$ is uniquely identified by $x$. Analogously, $f$ is said to be $y$-measurable whenever $f(x, y)=f\left(x^{\prime}, y\right)$, for all $x, x^{\prime} \in \mathbb{R}$. Then, we will write, $f_{2}(y)=f(x, y)$.

Lemma 3. Let $f(x, y)$ be an $x$-measurable function. Then $f^{s w}$ is $y$-measurable and

$$
\begin{aligned}
\underline{\mathbb{E}}_{\Gamma_{X Y}}(f) & =\underline{\mathbb{E}}_{\pi_{X}}\left(f_{1}\right) \\
\underline{\mathbb{E}}_{\Gamma_{X Y}}\left(f^{s w}\right) & =\underline{\mathbb{E}}_{\pi_{Y}}\left(f_{2}^{s w}\right) \\
\overline{\mathbb{E}}_{\Gamma_{X Y}}(f) & =\overline{\mathbb{E}}_{\pi_{x}}\left(f_{1}\right) \\
\underline{\mathbb{E}}_{\Gamma_{X Y}}\left(f^{s w}\right) & =\overline{\mathbb{E}}_{\pi_{Y}}\left(f_{2}^{s w}\right)
\end{aligned}
$$

Proof. We will only show the first equality, as the others can be derived with analogous reasoning. First note that the value $\underline{\mathbb{E}}_{\pi_{X}}(f)$ is well defined when $f(x, y)$ is $x$-measurable. We have that

$$
\begin{aligned}
\underline{\mathbb{E}}_{\Gamma_{X Y}}(f) & =\int_{[0,1]^{2}} \inf _{\substack{x \in \pi_{X, \alpha} \\
y \in \pi_{Y, \alpha}}} f(x, y) d P(\alpha, \beta)=\int_{[0,1]^{2}} \inf _{x \in \pi_{X, \alpha}} f_{1}(x) d P(\alpha, \beta) \\
& =\int_{[0,1]} \inf _{x \in \pi_{X, \alpha}} f_{1}(x) d P(\alpha)=\underline{\mathbb{E}}_{\pi_{X}}(f)
\end{aligned}
$$

with the last equality due to the fact that $P$ is the uniform distribution on $[0,1]$.

In particular, Lemma 3 indicates that when $f$ is $x$-measurable, the values of intervals $\left[\underline{\mathbb{E}}_{f, \mathrm{dep}}, \overline{\mathbb{E}}_{f, \mathrm{dep}}\right]$ and $\left[\underline{\mathbb{E}}_{f s w}, \mathrm{dep}, \overline{\mathbb{E}}_{f}{ }^{s w}, \mathrm{dep}\right]$ do not depend on the chosen dependence, hence neither does the relation $\geq_{f \text {,dep,ord }}$ derived from them. Also, computing $\left[\underline{\mathbb{E}}_{\Gamma_{X Y}}(f), \overline{\mathbb{E}}_{\Gamma_{X Y}}(f)\right]$ becomes straightforward by using Equations (8), as shown in the next corollary of Proposition 1

Corollary 4. Let $f(x, y)$ be an $x$-measurable function. Then

- $\underline{\mathbb{E}}_{\Gamma_{X Y}}(f)=\underline{\mathbb{E}}_{\pi_{X}}\left(f_{1}\right)-\overline{\mathbb{E}}_{\pi_{Y}}\left(f_{2}^{s w}\right)$

- $\overline{\mathbb{E}}_{\Gamma_{X Y}}(f)=\overline{\mathbb{E}}_{\pi_{X}}\left(f_{1}\right)-\underline{\mathbb{E}}_{\pi_{Y}}\left(f_{2}^{s w}\right)$.

\section{Fuzzy ranking as an imprecise probabilistic problem}

In this section, we explore how the tools of the previous sections can be applied to fuzzy intervals and the problem of fuzzy rankings. We explore the three classical ways to stochastically compare random variables, namely statistical preference (Sections 4.1 and 4.2), stochastic dominance (Section 4.3) and expectation-based preference (Section 4.4. 


\subsection{Statistical preference extension}

A random variable $X$ is said to be statistically preferred [36] to another random variable $Y$ when it satisfies the inequality $P(X>Y) \geq P(Y>X)$. Equivalently, and according to [10], we can say that $X$ is statistically preferred to $Y$ if $E\left[g_{>}(X, Y)\right] \geq 0$, where $g_{>}: \mathbb{R}^{2} \rightarrow \mathbb{R}$ is defined as follows:

$$
g_{>}(x, y)=\operatorname{sign}(x-y)=\left\{\begin{aligned}
1 & \text { if } x>y \\
0 & \text { if } x=y \\
-1 & \text { if } x<y
\end{aligned}\right.
$$

and $g_{>}$can be expressed as $g_{>}=f_{>}-f_{>}^{s w}$, where $f_{>}$is defined by

$$
f_{>}(x, y)=\left\{\begin{array}{cc}
1 & \text { if } x>y \\
0 & \text { else. }
\end{array}\right.
$$

Every criterion considered in this subsection will particularize the general Equation (21) for $f=f_{>}$, according to some particular dependence assumption dep $\in\{M, I, A M\}$ and some specific ordering between intervals, ord $\in\{w o, M m, M M, H(\lambda), I D, L o\}$. We will only detail some of them as illustrations, as the other relations and orderings can be obtained by simply applying the definitions of Section 3.2 for any choice of dep, ord.

To avoid making long lists of similar equations, we will only provide some details for the monotonic dependence case, formulas for other dependence assumptions easily following by replacing the integrals within the formulas. Under the monotonic dependence, we can extend interval ordering in the following way:

$$
X \geq_{f_{>}, M, I D} Y \quad \text { if and only if } \quad \int_{0}^{1} \inf _{\substack{x \in \pi_{X, \alpha} \\ y \in \pi_{Y, \alpha}}} f_{>}(x, y) d \alpha \geq \int_{0}^{1} \sup _{\substack{x \in \pi_{X, \alpha} \\ y \in \pi_{Y, \alpha}}} f_{>}^{s w}(x, y) d \alpha
$$

or equivalently if

$$
\int_{0}^{1} \mathbf{1}_{\left(\underline{\pi}_{X, \alpha}>\bar{\pi}_{Y, \alpha}\right)} d \alpha \geq \int_{0}^{1} \mathbf{1}_{\left(\bar{\pi}_{Y, \alpha}>\underline{\pi}_{X, \alpha}\right)} d \alpha
$$

where $\mathbf{1}_{(A)}$ is the indicator function of some event $A$, i.e., $\mathbf{1}_{(A)}=1$ if $A$ is true, 0 otherwise. The above formula comes down to apply interval dominance to each $\alpha$ cut, and is equivalent to comparing the lower probability $\underline{P}_{M}(X>Y)$ and the upper probability $\bar{P}_{M}(Y>X)$, under monotonic dependence, of the respective events $X>Y$ and $Y>X$. Proposition 1 tells us that Equation 24) can be alternatively written in terms of $g_{>}$as follows:

$$
X \geq_{f_{>}, M, I D} Y \quad \text { if and only if } \quad \int_{0}^{1} \inf _{\substack{x \in \pi_{X, \alpha} \\ y \in \pi_{Y, \alpha}}} g_{>}(x, y) d \alpha \geq 0 .
$$

This equation fits with the generalization of statistical preference considered in [12].

Replacing the infimum by the supremum in formula (26), we would get the upper expectation of $\mathbf{1}_{(X>Y)}-\mathbf{1}_{(Y>X)}$, giving the criterion

$$
\int_{0}^{1} \sup _{\substack{x \in \pi_{X, \alpha} \\ y \in \pi_{Y, \alpha}}} g_{>}(x, y) d \alpha \geq 0 \quad \text { if and only if } \quad X \geq_{f_{>}, M, w o} Y,
$$


where the equivalence with $\geq_{f_{>}, M, w o}$ follows from Proposition 1 . Equivalently, we have $X \geq_{>, M, w o} Y$ iff

$$
\int_{0}^{1} \sup _{\substack{x \in \pi_{X, \alpha} \\ y \in \pi_{Y, \alpha}}} f_{>}(x, y) d \alpha \geq \int_{0}^{1} \inf _{\substack{x \in \pi_{X, \alpha} \\ y \in \pi_{Y, \alpha}}} f_{>}^{s w}(x, y) d \alpha
$$

or iff

$$
\int_{0}^{1} \mathbf{1}_{\left(\bar{\pi}_{X, \alpha}>\underline{\pi}_{Y, \alpha}\right)} d \alpha \geq \int_{0}^{1} \mathbf{1}_{\left(\underline{\pi}_{Y, \alpha}>\bar{\pi}_{X, \alpha}\right)} d \alpha .
$$

This is a very weak criterion to compare fuzzy sets. Actually the left hand side in Equation (29) corresponds to the upper probability of the event $X>Y$, while the right hand side corresponds to the lower probability of $Y>X$, under monotonic dependence.

The previous remark indicates that $\left[\underline{\mathbb{E}}_{f_{>}, M}, \overline{\mathbb{E}}_{f_{>}, M}\right]=\left[\underline{P}_{M}(X>Y), \bar{P}_{M}(X>Y)\right]$ and $\left[\underline{\mathbb{E}}_{f_{>}^{s w}, M}, \overline{\mathbb{E}}_{f_{>}^{s w}, M}\right]=\left[\underline{P}_{M}(Y>X), \bar{P}_{M}(Y>X)\right]$, therefore all other criteria comes down to compare these lower/upper probabilities in specific ways. For instance, Maximin and Maximax criteria read

$$
\begin{array}{lll}
X \geq_{f_{>}, M, M m} Y & \text { if and only if } & \underline{P}_{M}(X>Y) \geq \underline{P}_{M}(Y>X), \\
X \geq_{f_{>}, M, M M} Y & \text { if and only if } & \bar{P}_{M}(X>Y) \geq \bar{P}_{M}(Y>X) .
\end{array}
$$

The extension of the lattice ordering then corresponds to

$$
X \geq_{f_{>}, M, L o} Y \quad \text { if and only if } \quad X \geq_{f_{>}, M, M m} Y \text { and } X \geq_{f_{>}, M, M M} .
$$

Finally, the Hurwicz criterion with parameter $\gamma$ can be extended by

$$
\begin{gathered}
X \geq_{f_{>}, M, H(\gamma)} Y \\
\text { if and only if } \\
\underline{\gamma}_{M}(X>Y)+(1-\gamma) \bar{P}_{M}(X>Y) \geq \gamma \underline{P}_{M}(Y>X)+(1-\gamma) \bar{P}_{M}(Y>X) .
\end{gathered}
$$

Similar equations can be obtained by replacing the dependence $M$ by either $A M$ or $I$, and by considering the corresponding formula among Equations (15)-(20).

\subsection{An alternative extension of statistical preference}

If $X$ and $Y$ are two random variables that satisfy the condition $P(X=Y)=0$ (e.g., when their joint distribution is continuous), then $X$ is statistically preferred to $Y$ when $P(X \geq Y) \geq 0.5$, or in other words, when the expectation of $\mathbf{1}_{(X \geq Y)}$ w.r.t. $P$ is greater than or equal to 0.5 . We can propose alternative generalizations of statistical preference under the above assumption about $X$ and $Y$.

Such extensions will consist in comparing, for each pair $(\alpha, \beta)$, the intervals of values $\left[\underline{\pi}_{X, \alpha}, \bar{\pi}_{X, \alpha}\right]$ and $\left[\underline{\pi}_{Y, \beta}, \bar{\pi}_{Y, \beta}\right]$ by means of different interval orderings, and then calculating the expectation, with respect to $P$, of such comparisons, under the different (in)dependence assumptions of Subsection 2.2. Such an expectation results in a number between 0 and 1 . In order to make a decision, it is reasonable to compare it with 0.5 . 
This approach will consist in reformulating interval orderings under the different (in)dependence assumptions of Subsection 2.2 by using the indicator function

$$
f_{\geq}(x, y)=\left\{\begin{array}{cc}
1 & \text { if } x \geq y \\
0 & \text { else }
\end{array}\right.
$$

For each particular ordering ord $\in\{w o, M m, M M, H(\lambda), I D\}$ and each possible dependence assumption dep, we will get the following expectation:

$$
(X, Y)_{\mathrm{dep}, \geq_{\mathrm{ord}}}=\int_{[0,1]^{2}} f_{\geq}\left(x_{\alpha}^{\mathrm{ord}}, y_{\beta}^{\mathrm{ord}}\right) d P_{\mathrm{dep}}(\alpha, \beta),
$$

where $P_{\mathrm{dep}}$ is the probability measure on $[0,1]^{2}$ having uniform marginals corresponding to dep $\in\{A, A M, I\}$ and the pair $\left(x_{\alpha}^{\text {ord }}, y_{\beta}^{\text {ord }}\right)$ is calculated as follows, according to each particular interval ordering:

- Weak ordering: $x_{\alpha}^{w o}=\bar{\pi}_{X, \alpha}, y_{\beta}^{w o}=\underline{\pi}_{Y, \beta}$.

- Maximin: $x_{\alpha}^{M m}=\underline{\pi}_{X, \alpha}, y_{\beta}^{M m}=\underline{\pi}_{Y, \beta}$.

- Maximax: $x_{\alpha}^{M M}=\bar{\pi}_{X, \alpha}, y_{\beta}^{M M}=\bar{\pi}_{Y, \beta}$.

- Hurwicz: $x_{\alpha}^{H(\lambda)}=\lambda \underline{\pi}_{X, \alpha}+(1-\lambda) \bar{\pi}_{X, \alpha}, y_{\beta}^{H(\lambda)}=\lambda \underline{\pi}_{Y, \beta}+(1-\lambda) \bar{\pi}_{Y, \beta}$.

- Interval dominance: $x_{\alpha}^{I D}=\underline{\pi}_{X, \alpha}, y_{\beta}^{I D}=\bar{\pi}_{Y, \beta}$.

In the case of the lattice order, the expectation of our evaluations will be:

$$
\begin{aligned}
(X, Y)_{\mathrm{dep}, \geq_{\mathrm{Lo}}} & =\int_{[0,1]^{2}} \min \left(f_{\geq}\left(\underline{\pi}_{X, \alpha}, \underline{\pi}_{Y, \beta}\right), f_{\geq}\left(\bar{\pi}_{X, \alpha}, \bar{\pi}_{Y, \beta}\right)\right) d P_{\mathrm{dep}}(\alpha, \beta) \\
& =\int_{[0,1]^{2}} \min \left(f_{\geq}\left(x_{\alpha}^{M m}, y_{\beta}^{M m}\right), f_{\geq}\left(x_{\alpha}^{M M}, y_{\beta}^{M M}\right) d P_{\mathrm{dep}}(\alpha, \beta) .\right.
\end{aligned}
$$

Let us pay attention to some particular cases of Equation (35). Its formulation for interval ordering coincides with the preference criterion considered in [34], provided in a machine learning procedure based on low quality data. In particular, under the monotonic dependence, we can extend interval ordering in the following way:

$$
(X, Y)_{M, \geq I D}=\int_{0}^{1} \inf _{\substack{x \in \pi_{X, \alpha} \\ y \in \pi_{Y, \alpha}}} f_{\geq}(x, y) d \alpha=\int_{0}^{1} \mathbf{1}_{\left(\underline{\pi}_{X, \alpha} \geq \bar{\pi}_{Y, \alpha}\right)} d \alpha .
$$

The above formula comes down to apply interval dominance to each $\alpha$-cut, and is equivalent to computing the lower probability $\underline{P}_{M}(X \geq Y)$, under monotonic dependence, of the event $X \geq Y$. It is also interesting to note that $(X, Y)_{M, \geq_{I D}}=1$ if and only if $\underline{\pi}_{X, 0} \geq \bar{\pi}_{Y, 0}$, that is if the support of $\pi_{X}$ interval dominates the support of $\pi_{Y}$.

The same extension can be built using an independence notion, in this case, interval dominance is extended into

$$
(X, Y)_{I, \geq I D}=\int_{0}^{1} \int_{0}^{1} \inf _{\substack{x \in \pi_{X, \alpha} \\ y \in \pi_{Y, \beta}}} f_{\geq}(x, y) d \alpha d \beta=\int_{0}^{1} \int_{0}^{1} \mathbf{1}_{\left(\underline{\pi}_{X, \alpha} \geq \bar{\pi}_{Y, \beta}\right)} d \alpha d \beta .
$$



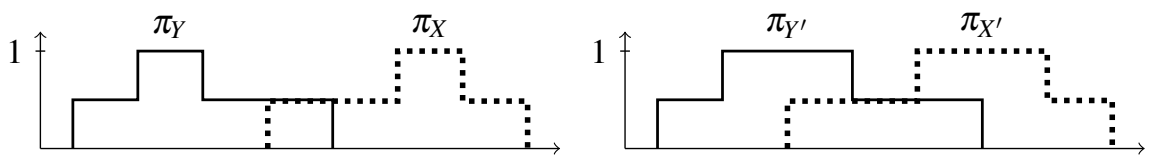

Figure 3: Two different cases where dependence assumption affects statistical preference extensions.

Equation (38) looks almost the same as 37), and still corresponds to the lower probability of $X \geq Y$, but under another dependence assumption. Note that, in general, we will have $(X, Y)_{I, \geq_{I D}} \neq(X, Y)_{M, \geq_{I D}}$, without one being always lower or higher than the other, as shown in Example 5 below. Again, we have that $(X, Y)_{I, \geq I D}=1$ if and only if $\underline{\pi}_{X, 0} \geq \bar{\pi}_{Y, 0}$.

Example 5. Consider the fuzzy sets pictured in Figure 3, for the two situations, we get the following values:

- $(X, Y)_{M, \geq_{I D}}=0.5$ and $(X, Y)_{I, \geq_{I D}}=0.75$

- $\left(X^{\prime}, Y^{\prime}\right)_{M, \geq_{I D}}=0.5$ and $\left(X^{\prime}, Y^{\prime}\right)_{I, \geq_{I D}}=0.25$

Again, we will not get into details about the rest of the possible generalizations of $P(X \geq Y)$ under different dependence assumptions, and different orderings between intervals, as they all can be written as particular cases of Equation (35). All of them provide gradual ways to compare two fuzzy sets, as they result in numbers ranging between 0 and 1 . Noticing that for any $\alpha, \beta \in[0,1]$ we have the following implications

$$
\underline{\pi}_{X, \alpha}>\bar{\pi}_{Y, \beta} \Rightarrow \begin{gathered}
\left(\underline{\pi}_{X, \alpha}>\underline{\pi}_{Y, \beta}\right) \\
\wedge \\
\left(\bar{\pi}_{X, \alpha}>\bar{\pi}_{Y, \beta}\right)
\end{gathered} \Rightarrow\left\{\begin{array}{c}
\underline{\pi}_{X, \alpha}>\underline{\pi}_{Y, \beta} \\
\bar{\pi}_{X, \alpha}>\bar{\pi}_{Y, \beta} \\
\gamma \underline{\pi}_{X, \alpha}+(1-\gamma) \pi_{X, \alpha}> \\
\gamma \underline{\pi}_{Y, \beta}+(1-\gamma) \bar{\pi}_{Y, \beta} \\
\Downarrow \\
\bar{\pi}_{X, \alpha}>\underline{\pi}_{Y, \beta}
\end{array} \text { for any } \gamma\right\}
$$

between the relation of $\alpha$-cut bounds, it follows that we have the following inequalities

$$
(X, Y)_{k, \geq_{I D}}<(X, Y)_{k, \geq_{L o}}<\left\{\begin{array}{l}
(X, Y)_{k, \geq_{M m}} \\
(X, Y)_{k, \geq_{M M}} \\
(X, Y)_{k, \geq_{H}(\gamma)}
\end{array}\right\}<(X, Y)_{k, \geq_{w o}}
$$

for any $k \in\{M, I D, A M\}$. These relations extend to gradual evaluations the relations existing between the interval comparisons introduced in Section 3.1 .

Also, we have that $(X, Y)_{k, \geq_{I D}}=\underline{P}(X \geq Y)$ and $(X, Y)_{k, \geq_{w o}}=\vec{P}(X \geq Y)$, meaning that

$$
\begin{aligned}
& X \geq_{f_{>}, I D, \text { dep }} Y \Rightarrow(X, Y)_{\mathrm{dep}, \geq I D} \geq 0.5, \\
& X \geq_{f_{>}, w o, \text { dep }} Y \Rightarrow(X, Y)_{\mathrm{dep}, \geq_{w o}} \geq 0.5,
\end{aligned}
$$

as $\underline{P}(X \geq Y) \geq \underline{P}(X>Y)$ and $\bar{P}(X \geq Y) \geq \bar{P}(X>Y)$. 
Yet, apart from the cases of interval dominance and weak ordering, there are no such clear and straightforward connections between the extensions of statistical preference proposed in Section 4.1 and those of Section 4.2. Indeed, consider for instance two distributions $\pi_{X}, \pi_{Y}$ such that $\pi_{X, 1} \cap \pi_{Y, 1} \neq \emptyset$ and $\pi_{Y}(c)=\pi_{X}(c+\delta)$ with $\delta>0$ $\left(\pi_{X}\right.$ is on the "right" of $\left.\pi_{Y}\right)$. In such a case, we have

$$
(X, Y)_{M, \geq M M}=\int_{0}^{1} \mathbf{1}_{\left(\bar{\pi}_{X, \alpha} \geq \bar{\pi}_{Y, \alpha}\right)} d \alpha=1 \quad \text { and } \quad(Y, X)_{M, \geq_{M M}}=0,
$$

clearly suggesting that $X$ is preferable to $Y$ according to maximax criterion of Section 4.2. Yet, we have $\left[\underline{P}_{M}(X>Y), \bar{P}_{M}(X>Y)\right]=[0,1]$ and $\left[\underline{P}_{M}(X<Y), \bar{P}_{M}(X<\right.$ $Y)]=[0,1]$ (simply consider that $\mathscr{P}\left(\Pi_{X}\right)$ and $\mathscr{P}\left(\Pi_{Y}\right)$ contains all Dirac measures located in $\pi_{X, 1}$ and $\pi_{Y, 1}$, respectively), clearly showing that

$$
X={ }_{f_{>}, M, M M} Y
$$

according to the criterion of Section 4.1, therefore reaching two quite different conclusions. The same example can be applied to the Maximin, Lattice ordering and Hurcwicz extensions.

As a final remark on this extension, note that the relation $(X, Y)_{\mathrm{dep}, \geq_{\text {ord }}}=1-$ $(Y, X)_{\text {dep }, \geq_{\text {ord }}}$ will not hold in general, unlike in the probabilistic setting (both values may be below 0.5 , for instance). Yet, for ord $\in\{I D, w o, M m, M M, l o\}$, we do have

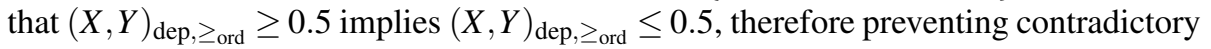
conclusions. To see that this is the case, simply note that for ord $\in\{I D, w o, M m, M M, l o\}$, if $\pi_{X, \alpha}>_{\text {ord }} \pi_{Y, \beta}$ then $\pi_{X, \alpha} \mathbb{Z}_{\text {ord }} \pi_{Y, \beta}$, which may not be the case for the weak ordering, that is too permissive.

\subsection{Stochastic dominance}

When quantities $X$ and $Y$ are modelled by cumulative distributions $F_{X}$ and $F_{Y}$, respectively, a classical way to compare them is through first-order stochastic dominance, that is to say that $X \succeq Y$ if and only if $F_{X}(c) \leq F_{Y}(c)$ for all $c \in \mathbb{R}$ or, equivalently, if $P(X>c) \geq P(Y>c)$, for all $c \in \mathbb{R}$. That is, $X$ stochastically dominates $Y$ if $X$ is more probable to have a value higher than $c$ than $Y$ is, for every $c \in \mathbb{R}$.

When the cumulative distributions of $X$ and $Y$ are bounded by a lower and an upper one, respectively $\left[\underline{F}_{X}, \bar{F}_{X}\right]$ and $\left[\underline{F}_{Y}, \bar{F}_{Y}\right]$, the notion of stochastic dominance $\succeq$ can again be extended in multiple ways [19, 32]. Such extensions are the result of applying Equation (21) to the family of mappings $\left\{f_{c}\right\}_{c \in \mathbb{R}}$, where $f_{c}: \mathbb{R}^{2} \rightarrow \mathbb{R}$ is defined as follows, for each $c \in \mathbb{R}$ :

$$
f_{c}(x, y)=\mathbf{1}_{(x>c)}, \forall(x, y) \in \mathbb{R}^{2} .
$$

Since $f_{c}$ is $x$-measurable, it means according to Lemma 3 that ranking notions derived from it do not rely on a particular dependence notion, meaning that if it holds for some choice of dep, it holds for any other. Now, if Equation 21) holds for all $c \in \mathbb{R}$ for some particular choice of ord, we denote the resulting relation by

$$
X \geq_{f_{\forall c}, \text { dep,ord }} Y \text {. }
$$


Extending stochastic dominance to convex sets of probabilities comes down to check stochastic dominance on specific pairs of cumulative functions [32], and we can associate them to ideas used in interval ordering:

- Weak ordering: $\left[\underline{F}_{X}, \bar{F}_{X}\right] \succeq_{w o}\left[\underline{F}_{Y}, \bar{F}_{Y}\right]$ if $\underline{F}_{X} \leq \bar{F}_{Y}$

- Maximin: $\left[\underline{F}_{X}, \bar{F}_{X}\right] \succeq_{M m}\left[\underline{F}_{Y}, \bar{F}_{Y}\right]$ if $\bar{F}_{X} \leq \bar{F}_{Y}$

- Maximax: $\left[\underline{F}_{X}, \bar{F}_{X}\right] \succeq_{M M}\left[\underline{F}_{Y}, \bar{F}_{Y}\right]$ if $\underline{F}_{X} \leq \underline{F}_{Y}$

- Hurwicz: $\left[\underline{F}_{X}, \bar{F}_{X}\right] \succeq_{H(\gamma)}\left[\underline{F}_{Y}, \bar{F}_{Y}\right]$ if $\gamma \bar{F}_{X}+(1-\gamma) \underline{F}_{X} \leq \gamma \bar{F}_{Y}+(1-\gamma) \underline{F}_{Y}$

- Interval dominance: $\left[\underline{F}_{X}, \bar{F}_{X}\right] \succeq_{I D}\left[\underline{F}_{Y}, \bar{F}_{Y}\right]$ if $\bar{F}_{X} \leq \underline{F}_{Y}$

- Lattice order: $\left[\underline{F}_{X}, \bar{F}_{X}\right] \succeq_{L o}\left[\underline{F}_{Y}, \bar{F}_{Y}\right]$ if $\bar{F}_{X} \leq \bar{F}_{Y}$ and $\underline{F}_{X} \leq \underline{F}_{Y}$

If a p-box is $\{0,1\}$ valued, that is corresponds to Dirac measures between a lower and an upper bounds, those extensions coincide with the interval comparisons bearing the same name (already in [32]). Additionally, implications (9) directly extend to the above extensions of first-order stochastic dominance. It is also easy to see that we have

$$
\left[\underline{F}_{X}, \bar{F}_{X}\right] \succeq_{k}\left[\underline{F}_{Y}, \bar{F}_{Y}\right] \Leftrightarrow X \geq_{f_{\forall c}, \text { dep }, k} Y
$$

for $k \in\{w o, M m, M M, H(\gamma), I D, L o\}$ and with any dependence, since $f_{c}$ is an $x$-measurable function (see Lemma 3 .

These comparisons are all-or-nothing comparisons. However, if we consider the p-boxes $\left[\underline{F}_{\pi_{X}}, \bar{F}_{\pi_{X}}\right]$ and $\left[\underline{F}_{\pi_{Y}}, \bar{F}_{\pi_{Y}}\right]$, we can relate these comparisons with extensions of interval comparisons proposed in Section 3.1. First note that we have $\underline{F}_{\pi}^{-1}(\alpha)=\bar{\pi}_{1-\alpha}$ and $\bar{F}_{\pi}^{-1}(\alpha)=\underline{\pi}_{\alpha}$ where $F^{-1}$ denotes the generalized inverse distribution function. We then have the following relations:

- $X \geq_{f_{\forall c}, \text { dep }, w o} Y$ if and only if $(X, Y)_{A M, \geq_{w o}}=1$.

- $X \geq_{f_{\forall c}, \text { dep }, M m} Y$ if and only if $(X, Y)_{M, \geq_{M m}}=1$.

- $X \geq_{f_{\forall c}, \text { dep }, M M} Y$ if and only if $(X, Y)_{M, \geq_{M M}}=1$.

- $X \geq_{f_{\forall c}, \mathrm{dep}, H(\gamma)} Y$ if and only if $(X, Y)_{A M, \geq_{H(\gamma)}}=1$.

- $X \geq_{f_{\forall c}, \operatorname{dep}, I D} Y$ if and only if $(X, Y)_{A M, \geq_{I D}}=1$.

- $X \geq_{f_{\forall c}, \text { dep }, L o} Y$ if $(X, Y)_{M, \geq_{L o}}=1$.

This indicates that some of the evaluations explored in Section 4.2 can be seen as gradual versions of stochastic dominance extensions (with them being equal to 1 if and only if the corresponding stochastic dominance holds). This also clarifies the assumptions that can be associated to the various extensions of stochastic dominance when applied to fuzzy intervals. In particular, it may be surprising at first to see that, while stochastic dominance notions do not depend on particular dependence notions, their relations with extensions of statistical preferences (Section 4.2) are linked to specific 
dependence assumptions (i.e., extensions of weak ordering, Hurwicz and interval dominance relate to anti-monotonic dependence, while the others relate to the monotonic dependence). Indeed, while the all-or-nothing relations do not depend on the dependence assumption, their gradual counterparts studied in Section 4.2 do, due to the fact that $\underline{F}_{\pi}^{-1}(\alpha)=\bar{\pi}_{1-\alpha}$ (therefore reversing the order of $\alpha$-cuts) and $\bar{F}_{\pi}^{-1}(\alpha)=\underline{\pi}_{\alpha}$.

\subsection{Expectation-based criteria}

So far, we have been mainly interested in qualitative comparisons, mainly checking whether or not some values were higher than others (using the indicator functions $f_{\geq}$ in various ways, or comparing cumulative distributions through function $f_{\forall c}$ ). One advantage of such formulations is that they only require values $x$ and $y$ to be comparable, i.e. they can be extended directly without much difficulty to arbitrary fuzzy sets defined on totally (pre-)ordered spaces. However, they are unable to measure "how far" fuzzy sets are from each other.

Another classical way to compare two (precise) random variables $X$ and $Y$ is to compare their expected values, stating that $X$ is higher than (preferred to) $Y$ if $\mathbb{E}(X) \geq$ $\mathbb{E}(Y)$. Expectations in our case can be modelled by choosing the function $f_{\mathbf{x}}: \mathbb{R}^{2} \rightarrow \mathbb{R}$ defined as $f_{\mathbf{x}}(x, y)=x, \forall(x, y) \in \mathbb{R}^{2}$. We can then consider the following orderings:

- Weak ordering: $X \geq_{f_{\mathbf{x}} \text {,dep,wo }} Y$ if $\overline{\mathbb{E}}(X) \geq \underline{\mathbb{E}}(Y)$,

- Maximin: $X \geq_{f_{\mathbf{x}}, \mathrm{dep}, M m} Y$ if $\underline{\mathbb{E}}(X) \geq \underline{\mathbb{E}}(Y)$,

- Maximax: $X \geq_{f_{\mathbf{x}}, \text { dep }, M M} Y$ if $\overline{\mathbb{E}}(X) \geq \overline{\mathbb{E}}(Y)$,

- Hurwicz: $X \geq_{f_{\mathbf{x}}, \mathrm{dep}, H(\gamma)} Y$ if $\gamma \underline{\mathbb{E}}(X)+(1-\gamma) \overline{\mathbb{E}}(X) \geq \gamma \mathbb{E}(Y)+(1-\gamma) \overline{\mathbb{E}}(Y)$,

- Interval dominance: $X \geq_{f_{\mathbf{x}}, \operatorname{dep}, I D} Y$ if $\underline{\mathbb{E}}(X) \geq \overline{\mathbb{E}}(Y)$,

- Lattice order: $X \geq_{f_{\mathbf{x}}, \mathrm{dep}, L o} Y$ if $\overline{\mathbb{E}}(X) \geq \overline{\mathbb{E}}(Y)$ and $\underline{\mathbb{E}}(X) \geq \underline{\mathbb{E}}(Y)$,

that are valid whatever the dependence assumption dep, since $f_{\mathbf{X}}$ is $x$-measurable (see Lemma 3). Again, implications (9) directly extend to these relations.

When dealing with imprecise probabilities (in our case, modelled by possibility distributions), the maximality criterion [41, 39] consists in computing the lower expectation $\underline{\mathbb{E}}(X-Y)$, and to say that $X$ is higher than (preferred to) $Y$ if $\mathbb{E}(X-Y) \geq 0$. Usually, we have that $\underline{\mathbb{E}}(X-Y)>\underline{\mathbb{E}}(X)+\underline{\mathbb{E}}(-Y)=\underline{\mathbb{E}}(X)+\overline{\mathbb{E}}(Y)$, hence $X \geq_{f_{\mathbf{x}} \text {,dep }, I D} Y$ implies $\underline{\mathbb{E}}(X-Y) \geq 0$, and not the other way around. However Corollary 4 indicates that in our framework they coincide. This result completes another one provided in [16], where the author states that both criteria do coincide under some independence assumptions including the so-called epistemic irrelevance, epistemic independence, strong independence or Kuznetsov independence. We do not assume any particular (in)dependence condition, but on the other side, we implicitly assume that the joint lower probability is an $\infty$-monotone capacity (the lower probability, in the sense of Dempster [18] associated to the random set $\left.\Gamma_{X Y}\right)$. We can derive a similar result for the upper expectation $\overline{\mathbb{E}}(X-Y)$ from Corollary 4 , showing that the difference $\overline{\mathbb{E}}(X)-\underline{\mathbb{E}}(Y)$ is positive when and only when $X \geq_{f_{\mathbf{x}} \text {,dep,wo }} Y$. Again, we can see that weak-ordering is really permissive, and will usually lead to a lot of indifference statements. 
When modelling uncertainty on $X$ and $Y$ by fuzzy intervals, lower/upper expectations take very the simple forms of Equations $(7)$, and $\underline{\mathbb{E}}(X), \overline{\mathbb{E}}(X)$ are simply the mean values of $\bar{F}_{\pi_{X}}$ and $\underline{F}_{\pi_{X}}$, respectively. Connecting this fact with the orderings proposed in Section 4.3, we get that

$$
X \geq_{f_{\forall c}, \mathrm{dep}, k} Y \Rightarrow X \geq_{f_{\mathbf{x}} \text {,dep }, k} Y
$$

for any $k \in\{w o, M m, M M, H(\gamma), I D, L o\}$, meaning that when comparing fuzzy intervals, statistical comparisons are actually stronger requirements than expectation based comparisons. Additionally, this result indicates a strong consistency with the precise setting, in which $X$ stochastically dominating $Y$ implies that $\mathbb{E}(u(X)) \geq \mathbb{E}(u(Y))$ for any increasing function $u$ [28]. Indeed, if $X \geq_{f_{\forall c} \text {,dep, },} Y$, then $u(X) \geq_{f_{\mathbf{x}} \text {,dep }, k} u(Y)$ for any $k \in\{w o, M m, M M, H(\gamma), I D, L o\}$, since the upper and lower expectations of $u(X), u(Y)$ would be computed using the cumulative distributions $\bar{F}_{\pi_{X}}, \underline{F}_{\pi_{X}}, \bar{F}_{\pi_{Y}}$ and $\underline{F}_{\pi_{Y}}$.

\section{Reinterpreting some classical fuzzy ranking methods}

Now that we have extensively studied the various ways in which stochastic orderings can be extended to fuzzy sets, we can look at the connections between such orderings and classical rankings of the fuzzy literature. We show that some of those fuzzy ranking indices actually coincide with some of the proposals made in the previous sections. However, it should be noted that most fuzzy ranking methods seek to provide complete (pre-)order between fuzzy intervals, without admitting incomparability. Usually, this is done either by summarizing the fuzzy interval to a single number (e.g., its expected value, its centroid), and the ranking is then obtained by comparing these numbers, or by thresholding some gradual measure $(X, Y) \in[0,1](X$ is then said higher than $Y$ if $(X, Y) \geq \tau$, where $\tau$ is some threshold, usually above 0.5$)$.

Note that our purpose is not to perform an extensive review of existing fuzzy rankings, as such reviews are already available [5, 42, 43]. We also decided to include only those fuzzy rankings that had a clear link with the imprecise probabilistic approach: other fuzzy ranking methods could perhaps be given a complex imprecise probabilistic interpretation, however this goes beyond the scope of this paper. For instance, the approach of Yager et al. [45] that consists in using statistical preference induced by the independent probability distributions $p_{X}(x)=\pi_{X}(x) / \Sigma_{x^{\prime}} \pi_{X}\left(x^{\prime}\right)$ and $p_{Y}(y)=\pi_{Y}(y) / \Sigma_{y^{\prime}} \pi_{Y}\left(y^{\prime}\right)$, yet we may have that $p_{X} \notin \mathscr{P}\left(\Pi_{X}\right)$ and $p_{Y} \notin \mathscr{P}\left(\Pi_{Y}\right)$, hence its links with the current view are unclear (if any). A similar argument can be advanced for methods that are based on areas under distributions $\pi_{Y}, \pi_{X}$ and their combinations, such as the one of Nakamura [33].

\subsection{A controversial case revisited}

Figure 4 shows a controversial case of three fuzzy intervals, initially proposed in [5]. All authors agree that $X_{3}$ should not be ranked higher than $X_{2}$, who should not be ranked higher than $X_{1}$. However, some authors have argued that $X_{1}>X_{2}>X_{3}$ was the most natural ranking [44, 8], while others have argued that $X_{1}, X_{2}$ and $X_{3}$ 


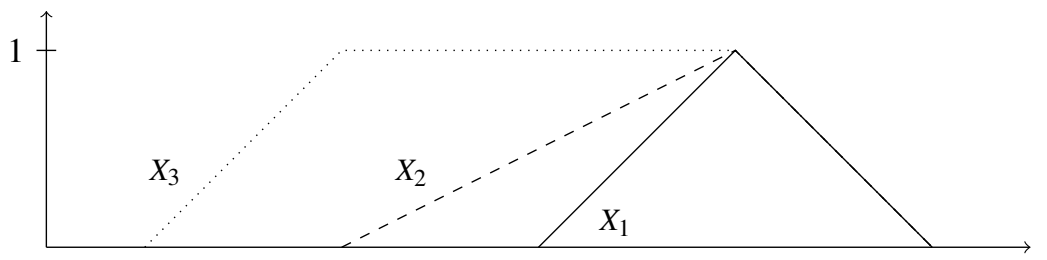

Figure 4: A controversial case

should be considered indistinguishable [3, 1], that is the ranking $X_{1} \sim X_{2} \sim X_{3}$ should be retained.

It is worth noticing that both rankings are coherent with particular orderings studied in the previous sections. When considering monotonic dependence, orderings extending lattice ordering, Hurwicz criterion with $\gamma \neq 0$ or maximin approaches will result in $X_{1}>X_{2}>X_{3}$, while orderings extending maximax or weak ordering will result in $X_{1} \sim X_{2} \sim X_{3}$. Under anti-monotonic dependence and random set independence, $\left(X_{i}, X_{j}\right)_{\text {dep,ord }}$ will take different values in $[0,1]$ (we leave the exact computations to the reader), with the value being in $\{0,1\}$ under monotonic dependence (dep $=M$ ).

The stronger requirements extending interval dominance will result in all fuzzy sets $X_{1}, X_{2}, X_{3}$ being incomparable.

\subsection{Dubois and Prade ranking}

Given two possibility distributions $\pi_{X}, \pi_{Y}$, Dubois and Prade [22] propose four different numbers to characterize whether one distribution is higher than the other. These numbers are defined as follows:

$$
\begin{gathered}
P D(X, Y)=\operatorname{supinf}_{x \geq y}\left(\pi_{X}(x), \pi_{Y}(y)\right), \\
N S D(X, Y)=1-\operatorname{supinf}_{x \leq y}\left(\pi_{X}(x), \pi_{Y}(y)\right), \\
P S D(X, Y)=\sup _{x} \inf _{x \leq y} \inf \left(\pi_{X}(x), 1-\pi_{Y}(y)\right), \\
N D(X, Y)=\inf _{x} \sup _{x \geq y} \inf \left(1-\pi_{X}(x), \pi_{Y}(y)\right) .
\end{gathered}
$$

Equation (41) is equivalent to the proposition made by Baas and Kwakernaak [3] in another paper. These equations can be rewritten as follows:

$$
\begin{gathered}
P D(X, Y)=\sup \left\{\alpha \mid \bar{\pi}_{X, \alpha} \geq \underline{\pi}_{Y, \alpha}\right\}, \\
N S D(X, Y)=1-\sup \left\{\alpha \mid \underline{\pi}_{X, \alpha} \leq \bar{\pi}_{Y, \alpha}\right\}=1-P D(Y, X), \\
P S D(X, Y)=\sup \left\{\alpha \mid \bar{\pi}_{X, \alpha} \geq \bar{\pi}_{Y, 1-\alpha}\right\}, \\
N D(X, Y)=\sup \left\{\alpha \mid \underline{\pi}_{X, 1-\alpha} \geq \underline{\pi}_{Y, \alpha}\right\} .
\end{gathered}
$$

The first relation is due to the fact that $P D(X, Y)=1$ if $\bar{\pi}_{X, 1} \geq \underline{\pi}_{Y, 1}$, and to the fact that $\underline{\pi}_{\alpha}, \bar{\pi}_{\alpha}$ are respectively increasing and decreasing functions of $\alpha$, hence if $\bar{\pi}_{X, 1}<\underline{\pi}_{Y, 1}$, the value of Equation (41) is reached at the intersection of the two functions $\bar{\pi}_{X, \alpha}$ and 
$\underline{\pi}_{Y, \alpha}$. Similarly, $P S D(X, Y)=1$ if $\bar{\pi}_{X, 1} \geq \bar{\pi}_{Y, 0}$, and if $\bar{\pi}_{X, 1}<\bar{\pi}_{Y, 0}$, then Equation (47) can be deduced from the fact that $\bar{\pi}_{\alpha}$ and $\bar{\pi}_{1-\alpha}$ are respectively decreasing and increasing functions of $\alpha$. Equations for $N S D$ and $N D$ can be obtained by duality.

There are some relation between Dubois and Prade ranking and our own relations:

Proposition 6. Given two distributions $\pi_{X}, \pi_{Y}$, the following equalities hold

- $P D(X, Y)=(X, Y)_{M, \geq w o}$,

- $N S D(X, Y)=(X, Y)_{M, \geq I D}$,

- $\operatorname{PSD}(X, Y)=(X, Y)_{A M, \geq_{M M}}$,

- $N D(X, Y)=(X, Y)_{A M, \geq_{M m}}$.

Proof. The first equality is immediate once we notice that:

- if $\bar{\pi}_{X, \alpha} \geq \underline{\pi}_{Y, \alpha}$ for some $\alpha$, then $\bar{\pi}_{X, \beta} \geq \underline{\pi}_{Y, \beta}$ for any $\beta \leq \alpha$ and

- if $\bar{\pi}_{X, \alpha} \leq \underline{\pi}_{Y, \alpha}$ for some $\alpha$, then $\bar{\pi}_{X, \beta} \leq \underline{\pi}_{Y, \beta}$ for any $\beta \geq \alpha$.

Meaning that $(X, Y)_{M, \geq_{w o}}=\sup \left\{\alpha \mid \bar{\pi}_{X, \alpha} \geq \underline{\pi}_{Y, \alpha}\right\}$. The equality between $\operatorname{NSD}(X, Y)$ and $(X, Y)_{M, \geq_{I D}}$ is obtained through duality of lower and upper probabilities, that is

$$
\begin{aligned}
N S D(X, Y) & =1-P D(Y, X)=1-(Y, X)_{M, \geq_{w o}}=1-\bar{P}_{M}(Y \geq X) \\
& =\underline{P}_{M}(X \geq Y)=(X, Y)_{M, \geq_{I D}} .
\end{aligned}
$$

The other equalities can be obtained by using reasoning similar to that of the first equality and considering an anti-monotonic dependence.

These results shed a new light over the rankings proposed by Dubois and Prade: if $P D(X, Y)$ and $N S D(X, Y)$ correspond to upper and lower probabilities of the event $X \geq$ $Y$ under the dependence assumption used in Zadeh's extension principle, $\operatorname{PSD}(X, Y)$ and $N D(X, Y)$ correspond to more unusual assumptions, extending the maximin and maximax approaches under an assumption of anti-monotonic dependence (this is due to the presence of $1-\pi_{Y}$ in Equations (43)-(44)). As argued by Dubois and Prade, when fuzzy sets reduce to crisp sets, we do find the four classical ways of ordering intervals. Yet from an imprecise probabilistic point of view, both $\operatorname{PSD}(X, Y)$ and $N D(X, Y)$ look less natural to use.

\subsection{Chanas et al. proposals}

Chanas et al. [6, 7] propose different comparison measures inspired by random set approaches. Basically, they propose eight probabilistic measures that consider either one random variable $A \sim \mathscr{U}_{[0,1]}$ uniformly distributed on $[0,1]$ or two independent 
random variables $A, B \sim \mathscr{U}_{[0,1]}$. The measures are defined as follows

$$
\begin{aligned}
& C Z_{1}(X, Y)=P\left(\underline{\pi}_{X, A} \geq \bar{\pi}_{Y, A}\right)=(X, Y)_{M, \geq_{I D}} \\
& C Z_{2}(X, Y)=P\left(\underline{\pi}_{X, A} \geq \bar{\pi}_{Y, B}\right)=(X, Y)_{I, \geq_{I D}} \\
& C Z_{3}(X, Y)=P\left(\underline{\pi}_{X, A} \geq \underline{\pi}_{Y, A}\right)=(X, Y)_{M, \geq_{M m}} \\
& C Z_{4}(X, Y)=P\left(\underline{\pi}_{X, A} \geq \underline{\pi}_{Y, B}\right)=(X, Y)_{I, \geq_{M m}} \\
& C Z_{5}(X, Y)=P\left(\bar{\pi}_{X, A} \geq \bar{\pi}_{Y, A}\right)=(X, Y)_{M, \geq_{M M}} \\
& C Z_{6}(X, Y)=P\left(\bar{\pi}_{X, A} \geq \bar{\pi}_{Y, B}\right)=(X, Y)_{I, \geq_{M M}} \\
& C Z_{7}(X, Y)=P\left(\bar{\pi}_{X, A} \geq \underline{\pi}_{Y, A}\right)=(X, Y)_{M, \geq_{w o}} \\
& C Z_{8}(X, Y)=P\left(\bar{\pi}_{X, A} \geq \underline{\pi}_{Y, B}\right)=(X, Y)_{I, \geq_{w o}}
\end{aligned}
$$

We have added the equalities with the relations explored in this paper, these inequalities simply resulting from the application of Equation 35. One thing we can notice is that in Sections 5.2 and 5.3 there is no fuzzy ranking score or extension that corresponds to relations extending the lattice order on intervals (while there are some extending the interval dominance order). One possible explanation is that lattice order relies on comparing pair of bounds, while the other approaches all focus on specific bounds of the $\alpha$-cuts.

\subsection{Campos and González approach}

In their paper [15], Campos and González propose to summarize a fuzzy set $\pi_{X}$ by the value $\mathbb{E}_{\beta}\left(\pi_{X}\right)$

$$
\mathbb{E}_{\beta}\left(\pi_{X}\right)=\int_{0}^{1} \frac{\beta \underline{\pi}_{X, \alpha}+(1-\beta) \underline{\pi}_{X, \alpha}}{2} d \alpha
$$

Clearly, we have

$$
\mathbb{E}_{\beta}\left(\pi_{X}\right)=\int_{0}^{1} \frac{\beta \underline{\pi}_{X, \alpha}}{2} d \alpha+\int_{0}^{1} \frac{(1-\beta) \underline{\pi}_{X, \alpha}}{2} d \alpha=\frac{\beta \underline{\mathbb{E}}(X)+(1-\beta) \overline{\mathbb{E}}(X)}{2}
$$

which comes down to use Hurwicz criterion on the expectation values with pessimism value $\beta$. Note that this proposal has also been made by Liou and Wang [30], and that the special case of $\beta=1 / 2$ has been proposed by many authors [44, 25, 46, 2] with different justifications.

To our knowledge, there are no existing proposal in the fuzzy literature corresponding to the extension of interval dominance, weak ordering and lattice ordering of lower and upper expectations.

Another popular way to summarize fuzzy intervals into numbers that can then be compared is the use of a so-called centroid, however this centroid (usually defined as the number $\left.C=\int x \pi_{X}(x) d x / \int \pi_{X}(x) d x\right)$ has not a clear relationship with an imprecise probabilistic view of fuzzy sets. Indeed, the (vertex) centroid of the set of probabilities $\mathscr{P}\left(\Pi_{X}\right)$ is the so-called pignistic probability distribution [37], whose expected value is $\underline{\mathbb{E}}(X)+\overline{\mathbb{E}}(X) / 2$ (or, in other words, Equation (57) with $\beta=1 / 2$ ). 


\section{Illustrative examples}

In this section, we provide the results of our ranking notions on examples similar to the ones used by Bortolan and Degani [5], in addition to the one already discussed in Section 5.1. In contrast with their study, we will not make a full analysis nor use all of their examples, as our purpose is illustrative. Also, we will only consider situations where fuzzy intervals are normalized (as said in Section 2) and where the cuts $\pi_{0}$ of the various fuzzy intervals are not disjoint (we have already discussed the fact that only extensions involving expected values can integrate how "far" fuzzy intervals are from each others).

Table 1 displays the details of the different proposed rankings for typical situations between two fuzzy sets. For statistical preferences, we focus on the monotone case in order to avoid displaying many pages of results. Clearly, all methods agree that in situations (a) and (c), $X$ is higher than $Y$, with the dominance in situation (c) being stronger (as show $\geq_{f_{x} \text {,dep, } I D}$ and $\geq_{f_{\forall c} \text {,dep }, I D}$ ).

The situation between (b) and (d) is more ambiguous, yet we can pinpoint an important difference: while (b) remains somehow ambiguous if we assume a pessimistic or an optimistic attitude, this is not the case of (d). This is patent if we look at minimax or maximax attitudes for all notions except for statistical preferences. It should however be noted that this difference between (b) and (d) appears differently in the various notions: while stochastic dominance declares $X$ and $Y$ incomparable for (b), the other notions (alternative statistical preference and expectation-based) consider them indifferent.

From all the notions, statistical preference appears to be the more conclusive, however this decisiveness also depends on the dependence. For instance, for situation (b) we do have $\left[\underline{P}_{A M}, \bar{P}_{A M}\right](X>Y)=[0,1]$ and $\left[\underline{P}_{A M}, \bar{P}_{A M}\right](Y>X)=[0,1]$, concluding indifference for statistical preference in this case.

\section{Conclusions}

In this paper, we have studied the problem of fuzzy ranking from an imprecise probabilistic perspective (something that, to our knowledge, has not been done before in such an extensive way). This has allowed us to introduce many possible rankings extending statistical and interval comparison tools. As such tools usually come with clear assumptions and interpretations, so do their extensions. Finally, we have related those rankings to classical fuzzy literature, thereby shedding new lights on old ranking techniques.

In our opinion, one of the main assets of interpreting fuzzy sets as imprecise probabilities is that, in this view, rankings with incomparability statements are natural options. Although such a property may not always be desirable, we think it may be useful to perform some tasks (i.e., deriving pareto front of uncertain classifiers, making cautious inferences and holding up the choice of a particular complete ranking, ...).

This first study defines rankings for fuzzy intervals describing uncertain quantities, and relates them to rankings proposed in the fuzzy literature when such relations are simple to establish. This fits the goal of the paper, which is to build some first bridge 
(a)

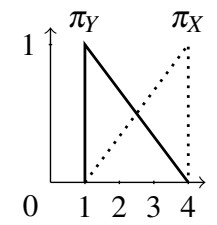

(b)

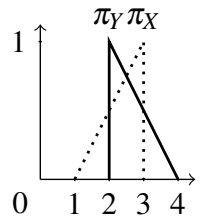

(c)

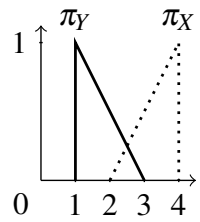

(d)

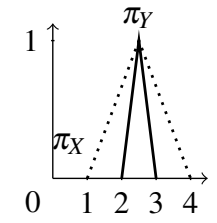

Statistical preference (Section 4.1)

\begin{tabular}{|c|c|c|c|c|}
\hline \\
\hline$\left[\underline{P}_{M}, \bar{P}_{M}\right](X>Y)$ & {$[0.5,1]$} & {$[0.25,1]$} & {$[0.75,1]$} & {$[0,1]$} \\
\hline$\left[\underline{P}_{M}, \bar{P}_{M}\right](Y>X)$ & {$[0,0.5]$} & {$[0,0.75]$} & {$[0,0.25]$} & {$[0,1]$} \\
\hline$\geq_{f_{>}, M, w o}$ & $X \geq Y$ & $X=Y$ & $X>Y$ & $X=Y$ \\
\hline$\geq f_{>}, M, M m$ & $X>Y$ & $X>Y$ & $X>Y$ & $X=Y$ \\
\hline$\geq_{f_{>}, M, M M}$ & $X>Y$ & $X>Y$ & $X>Y$ & $X=Y$ \\
\hline$\geq_{f_{>}, M, H(0.25)}$ & $X>Y$ & $X>Y$ & $X>Y$ & $X=Y$ \\
\hline$\geq_{f>, M, l o}$ & $X>Y$ & $X>Y$ & $X>Y$ & $X=Y$ \\
\hline$\geq_{f>M, I D}$ & $X \geq Y$ & $X><Y$ & $X>Y$ & $X><Y$ \\
\hline \multicolumn{5}{|c|}{ Alternative statistical preference (Section 4.2 ) } \\
\hline$(X, Y),(Y, X)_{M, w o}$ & $1,0.5$ & $1,0.75$ & $1,0.25$ & 1,1 \\
\hline$(X, Y),(Y, X)_{M, M m}$ & 1,0 & $0.5,0.5$ & 1,0 & 1,0 \\
\hline$(X, Y),(Y, X)_{M, M M}$ & 1,0 & $0.5,0.5$ & 1,0 & 0,1 \\
\hline$(X, Y),(Y, X)_{M, H(0.25)}$ & 1,0 & $0.5,0.5$ & 1,0 & 1,0 \\
\hline$(X, Y),(Y, X)_{M, l o}$ & 1,0 & $0.5,0.5$ & 1,0 & 0,0 \\
\hline$(X, Y),(Y, X)_{M, I D}$ & $0.5,0$ & $0.25,0$ & $0.75,0$ & 0,0 \\
\hline \multicolumn{5}{|c|}{ Stochastic dominance (Section 4.3 ) } \\
\hline$\geq_{f_{\forall c}, \mathrm{dep}, w o}$ & $X=Y$ & $X=Y$ & $X>Y$ & $X=Y$ \\
\hline$\geq_{f_{\forall c}, \operatorname{dep}, M m}$ & $X>Y$ & $X><Y$ & $X>Y$ & $X>Y$ \\
\hline$\geq f_{\forall c}, \operatorname{dep}, M M$ & $X>Y$ & $X><Y$ & $X>Y$ & $X<Y$ \\
\hline$\geq_{f_{\forall c}, \operatorname{dep}, H(0.25)}$ & $X>Y$ & $X><Y$ & $X>Y$ & $X>Y$ \\
\hline$\geq f_{\forall c}, \operatorname{dep}, l o$ & $X>Y$ & $X><Y$ & $X>Y$ & $X><Y$ \\
\hline$\geq f_{\forall c}, \mathrm{dep}, I D$ & $X \geq Y$ & $X><Y$ & $X>Y$ & $X><Y$ \\
\hline \multicolumn{5}{|c|}{ Expectation-based criteria (Section 4.3) } \\
\hline$[\underline{\mathbb{E}}(X), \overline{\mathbb{E}}(X)]$ & {$[2.5,4]$} & {$[2,3]$} & {$[3,4]$} & {$[2.25,2.75]$} \\
\hline$[\underline{\mathbb{E}}(Y), \overline{\mathbb{E}}(Y)]$ & {$[1,2.5]$} & {$[2,3]$} & {$[1,2]$} & {$[1.75,3.25]$} \\
\hline$\geq_{f_{x}, \mathrm{dep}, w o}$ & $X \geq Y$ & $X=Y$ & $X>Y$ & $X=Y$ \\
\hline$\geq_{f_{x}, \mathrm{dep}, M m}$ & $X>Y$ & $X=Y$ & $X>Y$ & $X>Y$ \\
\hline$\geq f_{x}$, dep,$M M$ & $X>Y$ & $X=Y$ & $X>Y$ & $X<Y$ \\
\hline$\geq_{f_{x}, \mathrm{dep}, H(0.25)}$ & $X>Y$ & $X=Y$ & $X>Y$ & $X>Y$ \\
\hline$\geq f_{x}, \mathrm{dep}, l o$ & $X>Y$ & $X=Y$ & $X>Y$ & $X><Y$ \\
\hline$\geq f_{x}, \operatorname{dep}, I D$ & $X \geq Y$ & $X><Y$ & $X>Y$ & $X><Y$ \\
\hline
\end{tabular}

Table 1: Behaviour of ranking for different situations 
between fuzzy rankings and imprecise probabilities. This also means that there are many ways in which we want to extend this bridge:

- by fully cataloguing the different fuzzy rankings and their interrelations, to then study which of them can be re-interpreted in an imprecise probabilistic setting (possibly using some new ways to rank imprecise probabilistic models);

- by studying various ways to transform the incomplete rankings (extending interval dominance and lattice ordering) into complete rankings, and how they connect to fuzzy rankings not considered in this study;

- by applying the ideas presented in this paper to applications involving comparisons of fuzzy sets (e.g., selection of solutions in evolutionary algorithms, construction of Pareto fronts, ....).

\section{Acknowledgments}

This work was partially carried out in the framework of the Labex MS2T (Reference ANR-11-IDEX-0004-02). It has been also partially supported by the Ministry of Economy and Competitiveness of the Spanish government under project TIN201124302.

\section{References}

[1] J. Adamo. Fuzzy decision trees. Fuzzy Sets and Systems, 4(3):207 - 219, 1980.

[2] B. Asady and A. Zendehnam. Ranking fuzzy numbers by distance minimization. Applied Mathematical Modelling, 31(11):2589 - 2598, 2007.

[3] S. Baas and H. Kwakernaak. Rating and Ranking of Multiple-Aspect Alternatives Using Fuzzy Setst. Automatica, 13:47-58, 1977.

[4] C. Baudrit and D. Dubois. Comparing methods for joint objective and subjective uncertainty propagation with an example in a risk assessment. In Proc. Fourth International Symposium on Imprecise Probabilities and Their Application (ISIPTA'05), pages 31-40, Pittsburg (USA, Pennsylvanie), 2005.

[5] G. Bortolan and R. Degani. A review of some methods for ranking fuzzy subsets. Fuzzy Sets and Systems, 15(1):1 - 19, 1985.

[6] S. Chanas, M. Delgado, J. Verdegay, and M. Vila. Ranking fuzzy interval numbers in the setting of random sets. Information Sciences, 69(3):201 - 217, 1993.

[7] S. Chanas and P. Zieliski. Ranking fuzzy interval numbers in the setting of random sets - further results. Information Sciences, 117(3-4):191-200, 1999.

[8] S.-H. Chen. Ranking fuzzy numbers with maximizing set and minimizing set. Fuzzy Sets and Systems, 17(2):113 - 129, 1985. 
[9] I. Couso. Independence concepts in evidence theory. In Proc. of the 5th Int. Symp. on Imprecise Probability: Theories and Applications, 2007.

[10] I. Couso and D. Dubois. An imprecise probability approach to joint extensions of stochastic and interval orderings. In Advances in Computational Intelligence, pages 388-399. Springer, 2012.

[11] I. Couso and D. Dubois. Statistical reasoning with set-valued information: Ontic vs. epistemic views. International Journal of Approximate Reasoning, 2014.

[12] I. Couso and L.Sánchez. The behavioral meaning of the median. In 5th International conference on soft methods in probability and statistics (SMPS 2010), Advances in Intelligent and Soft Computing, pages 115-122, Oviedo, 2010. Springer.

[13] I. Couso, S. Montes, and P. Gil. The necessity of the strong alpha-cuts of a fuzzy set. Int. J. on Uncertainty, Fuzziness and Knowledge-Based Systems, 9:249-262, 2001.

[14] I. Couso, S. Moral, and P. Walley. A survey of concepts of independence for imprecise probabilities. Risk Decision and Policy, 5:165-181, 2000.

[15] L. de Campos Ibáñez and A. González Muñoz. A subjective approach for ranking fuzzy numbers. Fuzzy Sets and Systems, 29(2):145-153, 1989.

[16] G. de Cooman. Further thoughts on possibilistic previsions: A rejoinder. Fuzzy Sets and Systems, 153:375-385, 2005.

[17] H. De Meyer, B. De Baets, and B. De Schuymer. On the transitivity of the comonotonic and countermonotonic comparison of random variables. Journal of Multivariate Analysis, 98(1):177-193, 2007.

[18] A. Dempster. Upper and lower probabilities induced by a multivalued mapping. Annals of Mathematical Statistics, 38:325-339, 1967.

[19] T. Denoeux. Extending stochastic ordering to belief functions on the real line. Information Sciences, 179(9):1362-1376, Apr. 2009.

[20] D. Dubois. The mean value of a fuzzy number. Fuzzy Sets and Systems, 24(3):279-300, 1987.

[21] D. Dubois. The role of fuzzy sets in decision sciences: Old techniques and new directions. Fuzzy Sets and Systems, 184(1):3-28, 2011.

[22] D. Dubois and H. Prade. Ranking fuzzy numbers in the setting of possibility theory. Information Sciences, 30(3):183 - 224, 1983.

[23] S. Ferson, L. Ginzburg, V. Kreinovich, D. Myers, and K. Sentz. Constructing probability boxes and dempster-shafer structures. Technical report, Sandia National Laboratories, 2003. 
[24] P. Fishburn. Interval Orderings. Wiley, New York, 1987.

[25] P. Fortemps and M. Roubens. Ranking and defuzzification methods based on area compensation. Fuzzy Sets and Systems, 82(3):319 - 330, 1996.

[26] J. Fürnkranz and E. Hüllermeier. Preference learning. Springer, 2010.

[27] M. Grabisch and C. Labreuche. A decade of application of the choquet and sugeno integrals in multi-criteria decision aid. Annals of Operations Research, 175(1):247-286, 2010.

[28] J. Hadar and W. R. Russell. Rules for ordering uncertain prospects. American Economic Review, 59:25-34, 1969.

[29] L. Hurwicz. A class of criteria for decision-making under ignorance. Cowles Comission Paper, 356, 1951.

[30] T.-S. Liou and M.-J. J. Wang. Ranking fuzzy numbers with integral value. Fuzzy Sets and Systems, 50(3):247 - 255, 1992.

[31] I. Montes, E. Miranda, and S. Montes. Decision making with imprecise probabilities and utilities by means of statistical preference and stochastic dominance. European Journal of Operational Research, 234(1):209-220, 2014.

[32] I. Montes, E. Miranda, and S. Montes. Stochastic dominance with imprecise information. Computational Statistics \& Data Analysis, 71:868-886, 2014.

[33] K. Nakamura. Preference relations on a set of fuzzy utilities as a basis for decision making. Fuzzy Sets and Systems, 20(2):147-162, 1986.

[34] N. Rescher and R. Manor. On inference from inconsistent premises. Theory and Decision, 1:179-219, 1970.

[35] L. E. Satia J.K., Roy J. Markovian decision processes with uncertain transition probabilities. Operations Research, 21(3):728-740, 1973.

[36] B. D. Schuymer, H. D. Meyer, B. D. Baets, and S. Jenei. On the cycle-transitivity of the dice model. Theory and Decision, 54:261-285, 2003.

[37] P. Smets. Decision making in the tbm: the necessity of the pignistic transformation. I.J. of Approximate Reasoning, 38:133-147, 2005.

[38] M. Sniedovich. Wald's maximin model: a treasure in disguise! Journal of Risk Finance, The 9(3):287-291, 2008.

[39] M. Troffaes. Decision making under uncertainty using imprecise probabilities. Int. J. of Approximate Reasoning, 45:17-29, 2007.

[40] A. Wald. Statistical decision functions which minimize the maximum risk. Annals of Mathematics, pages 265-280, 1945. 
[41] P. Walley. Statistical reasoning with imprecise Probabilities. Chapman and Hall, New York, 1991.

[42] X. Wang and E. E. Kerre. Reasonable properties for the ordering of fuzzy quantities (I). Fuzzy Sets and Systems, 118(3):375-385, 2001.

[43] X. Wang and E. E. Kerre. Reasonable properties for the ordering of fuzzy quantities (II). Fuzzy Sets and Systems, 118(3):387-405, 2001.

[44] R. R. Yager. A procedure for ordering fuzzy subsets of the unit interval. Inf. Sci., 24(2):143-161, 1981.

[45] R. R. Yager, M. Detyniecki, and B. Bouchon-Meunier. A context-dependent method for ordering fuzzy numbers using probabilities. Information Sciences, 138(1):237-255, 2001.

[46] J.-S. Yao and K. Wu. Ranking fuzzy numbers based on decomposition principle and signed distance. Fuzzy Sets and Systems, 116(2):275 - 288, 2000.

[47] L. Zadeh. Fuzzy sets as a basis for a theory of possibility. Fuzzy Sets and Systems, 1:3-28, 1978. 